\title{
RX J1548.9+0851, a fossil cluster?
}

\author{
P. Eigenthaler and W. W. Zeilinger
}

Institut für Astronomie, Universität Wien, Türkenschanzstraße 17, 1180 Vienna, Austria

e-mail: paul.eigenthaler@univie.ac.at

Received 8 December 2011 / Accepted 19 February 2012

\section{ABSTRACT}

\begin{abstract}
Context. Fossil galaxy groups are spatially extended X-ray sources with X-ray luminosities above $L_{\mathrm{X}}$,bol $\geq 10^{42} h_{50}^{-2} \mathrm{erg} \mathrm{s}^{-1}$ and a central elliptical galaxy dominating the optical, the second-brightest galaxy being at least 2 mag fainter in the $R$ band. Whether these systems are a distinct class of objects resulting from exceptional formation and evolution histories is still unclear, mainly due to the small number of objects studied so far, mostly lacking spectroscopy of group members for group membership confirmation and a detailed kinematical analysis.

Aims. To complement the scarce sample of spectroscopically studied fossils down to their faint galaxy populations, the fossil candidate RX J1548.9+0851 $(z=0.072)$ is studied in this work. Our results are compared with existing data from fossils in the literature.

Methods. We use ESO VLT VIMOS multi-object spectroscopy to determine redshifts of the faint galaxy population and study the luminosity-weighted dynamics and luminosity function of the system. The full-spectrum fitting package ULySS is used to determine ages and metallicities of group members. VIMOS imaging data are used to study the morphology of the central elliptical.

Results. We identify 40 group members spectroscopically within the central $\sim 300 \mathrm{kpc}$ of the system and find 31 additional redshifts from the literature, resulting in a total number of 54 spectroscopically confirmed group members within $1 \mathrm{Mpc}$. RX J1548.9+0851 is made up of two bright ellipticals in the central region with a magnitude gap of $\Delta m_{1,2}=1.34$ in the SDSS $r^{\prime}$ band leaving the definition of RX J1548.9+0851 being a fossil to the assumption of the virial radius. We find a luminosity-weighted velocity dispersion of $568 \mathrm{~km} \mathrm{~s}^{-1}$ and a mass of $\sim 2.5 \times 10^{14} M_{\odot}$ for the system confirming previous studies that revealed fossils to be massive. An average mass-to-light ratio of $M / L \sim 400 M_{\odot} / L_{\odot}$ is derived from the SDSS $g^{\prime}, r^{\prime}$, and $i^{\prime}$ bands. The central elliptical is well-fitted by a pure deVaucouleurs $r^{1 / 4}$ law without a cD envelope. Symmetric shells are revealed along the major axis of the galaxy indicating a recent minor merger. RX J1548.9+0851 shows a steep, increasing luminosity function with a faint-end slope of $\alpha=-1.4 \pm 0.1$. Satellite galaxies show a clear spatial segregation with respect to their stellar populations - objects with old stars are confined to an elongated, central distribution aligned with the major axis of the central elliptical.

Conclusions. Although RX J1548.9+0851 shows similar properties compared to other fossils studied recently, it might not be a fossil at all, being dominated by 2 bright central ellipticals. Comparing RX J1548.9+0851 with scaling relations from ordinary poor groups and clusters confirm the idea that fossils might simply be normal clusters with the richness and optical luminosity of poor groups.
\end{abstract}

Key words. galaxies: groups: individual: RX J1548.9+0851 - galaxies: elliptical and lenticular, cD - galaxies: luminosity function, mass function - galaxies: evolution - galaxies: distances and redshifts - galaxies: stellar content

\section{Introduction}

Jones et al. (2003) defined fossil galaxy groups to be spatially extended X-ray sources with X-ray luminosities above $L_{\mathrm{X}}$, bol $\geq$ $10^{42} h_{50}^{-2} \mathrm{erg} \mathrm{s}^{-1}$ and a central elliptical galaxy dominating the optical, all other galaxies within one-half virial radius being at least 2 mag fainter in the $R$ band. These observational characteristics suggested that fossils are the remnants of poor groups of galaxies that depleted their $L^{*}$ galaxies in the course of dynamical friction and merging, building up one massive central galaxy as predicted by numerical simulations (Barnes 1989). The first fossil was identified in 1994 (Ponman et al. 1994). Since then, several other objects have been assigned to this class (Vikhlinin et al. 1999; Jones et al. 2003; Yoshioka et al. 2004; Sun et al. 2004; Khosroshahi et al. 2004; Ulmer et al. 2005; Schirmer et al. 2010; Pierini et al. 2011; Miller et al. 2012). Santos et al. (2007) and Eigenthaler \& Zeilinger (2009) have cross-correlated optical and X-ray data from the Sloan Digital Sky Survey SDSS (Adelman-McCarthy et al. 2008) and the ROSAT All Sky Survey (Voges et al. 1999) to systematically identify new fossil candidates. Jones et al. (2003) estimated that fossil systems probably constitute $8-20 \%$ of all systems with comparable X-ray luminosity ( $\left.\geq 10^{42} \mathrm{erg} \mathrm{s}^{-1}\right)$, acting also very likely as the formation site of brightest cluster galaxies (BCGs). Similar optical luminosities of the central ellipticals in fossils compared to that of BCGs support this idea (Khosroshahi et al. 2006). The detection of these aggregates is challenging however, mainly because of their unremarkable appearance in the optical and the lack of high signal-to-noise ratio $(\mathrm{S} / \mathrm{N}) \mathrm{X}$-ray data.

Recent studies have shown that fossils are much more massive than what would be expected from the remnant of a groupsized halo, exhibiting velocity dispersions and X-ray temperatures comparable to poor clusters rather than groups (Mendes de Oliveira et al. 2006, 2009; Cypriano et al. 2006; Proctor et al. 2011), suppressing strong interactions between galaxies - inconsistent with the proposed merging scenario. Hence, there has been a lot of debate on the origin and formation scenario for fossil groups/clusters over the past few years. A few previous studies have investigated the scaling relations of known fossils in comparison to ordinary groups and clusters. Khosroshahi et al. (2007) concluded that fossils are overluminous in X-rays for a given optical luminosity, falling on the $L_{\mathrm{X}}-T_{\mathrm{X}}$ relation however. This excess in X-ray luminosity and temperature was interpreted by the presence of cuspy dark matter haloes suggesting 
an early formation epoch (Navarro et al. 1995). It was also suggested that fossils are not overluminous in X-rays but underluminous in the optical, being comparable to clusters in mass and $\mathrm{X}$-ray luminosity, but otherwise possessing the richness and optical luminosities of poor groups (Proctor et al. 2011). The missing baryons could have been expelled from the system, hidden in the intracluster light or were never present, suggesting that fossils formed in baryon deficient regions in space. Various studies also revealed that fossils exhibit comparatively high $M / L$ ratios with respect to ordinary systems (Vikhlinin et al. 1999; Yoshioka et al. 2004; Khosroshahi et al. 2007; Proctor et al. 2011).

Besides the observational effort to understand the formation and evolution of fossils, several simulations have been carried out addressing the same questions from a theoretical point of view. To trace the evolution of present day fossils back to their formation epoch, Dariush et al. (2007) made use of the Millennium and Millennium gas simulations (Springel et al. 2005; Hartley et al. 2008) revealing that at any given redshift, fossils have assembled a higher fraction of their final halo mass compared to non-fossils with similar X-ray luminosity. The latter systems assemble their halo mass even at the present day. These simulations suggest that fossils indeed formed early with most of their halo mass in place at higher redshifts. Using self consistent $N$-body/hydrodynamical simulations based on $\Lambda C D M$ cosmology, D’Onghia et al. (2005) found a similar result, a clear correlation between the $\Delta m_{1,2} \geq 2.0$ magnitude gap and the formation time of groups. The earlier a galaxy group is assembled, the larger the magnitude gap at $z=0$, suggesting that fossils have already assembled half of their final dark matter mass at $z \gtrsim 1$ and subsequently typically grow by minor mergers only. This early mass assembly leaves sufficient time for $L^{*}$ galaxies to be first tidally stripped and finally merge into the central elliptical by dynamical friction, resulting in the exceptional magnitude gap at $z=0$ whilst in non-fossils the final dark matter halo mass is assembled quite recently.

The overall motivation driving all these various observational and theoretical efforts aim at understanding whether fossils are a distinct class of objects resulting from a different formation and evolution history or being simply extreme examples of ordinary groups and clusters. The failure to give a clear explanation on the true nature of these aggregates is mainly due to the small number of objects studied so far, mostly lacking spectroscopy of group members for group membership confirmation and a detailed kinematical analysis.

To complement the scarce sample of spectroscopically studied fossils down to their faint galaxy populations, the fossil candidate RX J1548.9+0851 is studied in detail in this work using VIMOS MOS spectroscopy. The system was selected from the sample of Santos et al. (2007) based on its redshift and the high apparent brightness of the central elliptical. Assuming an X-ray temperature of $k T=2 \mathrm{keV}$ and a metallicity of $Z=0.4 Z_{\odot}$, Santos et al. (2007) estimate an X-ray luminosity of $L_{\mathrm{X}}=5.09 \times 10^{42} \mathrm{erg} \mathrm{s}^{-1}$ for the system from ROS AT count-rates. At a redshift of $z=0.0721$, the system lies at a luminosity distance of $D_{\mathrm{L}}=326 \mathrm{Mpc}$ and an angular diameter distance of $D_{\mathrm{A}}=283 \mathrm{Mpc}$ corresponding to a spatial scale of $1.374 \mathrm{kpc} \mathrm{arcsec}^{-1}$. At this redshift the age of the universe amounts to $\sim 12.5 \mathrm{Gyr}$.

The paper is organised as follows. In Sect. 2 observations and data analysis techniques are described whilst Sect. 3 presents all obtained results. Section 4 discusses and compares our findings with other fossils from the literature. Conclusions are given in Sect. 5. Magnitudes presented in this work are SDSS DR7 model magnitudes. Throughout the paper, the standard $\Lambda$ CDM cosmology with $\Omega_{M}=0.3, \Omega_{\Lambda}=0.7$, and a Hubble constant of $H_{0}=70 \mathrm{~km} \mathrm{~s}^{-1}$ is used.

\section{Observations and data reduction}

RX J1548.9+0851 was observed with the multi-object spectrograph VIMOS mounted on the ESO VLT UT3 in service mode. The observations were carried out in 2009 on February 25 for the pre-imaging and on April 24 and May 26 for the associated multi-object spectroscopy. VIMOS is made up of four individual quadrants, each with a field of view of $\sim 7 \times 8$ arcmin separated by gaps of 2 arcmin. The CCDs cover $4 \times 2048 \times 4096$ pixels with a pixel size of $15 \times 15 \mu$ m resulting in a spatial scale of 0.205 arcsec pixel ${ }^{-1}$. Considering the redshift of the system, one quadrant comprises roughly a radius of $300 \mathrm{kpc}$ on the sky. Thus, to cover essentially the inner regions of the group, a single pointing with the dominating elliptical centred on VIMOS quadrant 3 was scheduled. The pre-imaging was performed in the Bessel $R$ band and consisted of one $200 \mathrm{~s}$ exposure carried out under clear sky conditions with a measured seeing of $\sim 0.7$ arcsec FWHM on the images. For the further photometric analysis, the reduced data automatically preprocessed by the ESO reduction pipeline ${ }^{1}$ was considered. ESO photometric zeropoints were used to transform instrumental magnitudes to the standard magnitude system. Since zeropoints are given in ADU s ${ }^{-1}$, the reduced science frames were first divided by their exposure time. The resulting magnitudes were checked by comparing magnitudes of some bright stars in each quadrant with SDSS $r^{\prime}$ band model magnitudes. To obtain reliable radial velocity measurements, the VIMOS HR blue grism with a spectral resolution of $0.51 \AA$ pixel $^{-1}$ covering a wavelength range of 4150 to $6200 \AA$ was used. Spectroscopic targets were selected based on all SDSS sources in the VIMOS field brighter than $i^{\prime}=20$ mag that have been photometrically classified as galaxies. Obvious stars falsely classified as galaxies as well as objects in the immediate vicinity of the central elliptical hardly seen on the pre-image have not been considered for the target selection. To cover as many faint galaxies in the inner region of RX J1548.9+0851 as possible, two slit masks have been prepared for quadrant 3 . For brighter objects with larger spatial extent, slit-lengths were increased to a more suitable value. The spectroscopic observations were carried out under clear sky conditions and consisted of $4500 \mathrm{~s}$ exposures for every slit mask. The measured seeing in the spectra was around $\sim 0.8$ arcsec. In total, 97 slits were assigned in the whole observed VIMOS field, out of which 90 spectra have been extracted by the VIMOS pipeline $^{2}$. To check for remaining sky lines in the pipeline processed spectra, each spectrum was compared with the associated sky model. If a strong feature was present at a certain wavelength in the model and the object spectrum, it was deleted from the object spectrum manually within the IRAF splot task.

\footnotetext{
1 The pipeline does bias subtraction, overscan removal, flat-fielding and cosmic ray removal. Images are photometrically calibrated with photometric zeropoints, extinction coefficients, airmass, and an estimate of the sky background stored in the image header of each individual quadrant.

2 The method of Horne (1986) is used to extract one-dimensional spectra. The pipeline applies basic reduction steps (bias subtraction and wavelength calibration) and flux-calibrates the raw spectra. The flux calibration is relative instead of absolute, fluxes being valid except for a constant factor. A median sky level is estimated for each wavelength and subtracted from the data. The extracted spectra are resampled to a constant wavelength interval ( $0.603 \AA \mathrm{pix}^{-1}$ for the HR blue grism).
} 


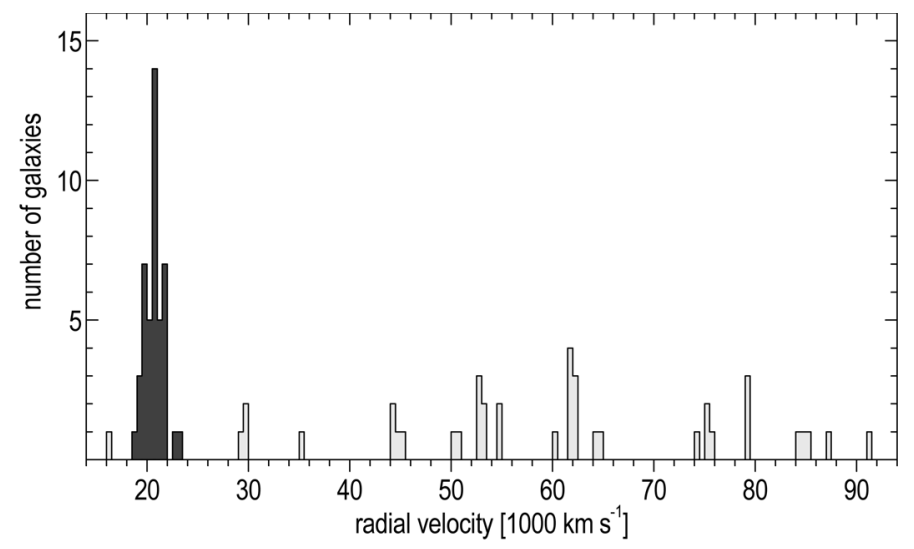

Fig. 1. Distribution of measured radial velocities for all galaxies in the observed VIMOS field. The velocity range covers $80000 \mathrm{~km} \mathrm{~s}^{-1}$ with a bin size of $500 \mathrm{~km} \mathrm{~s}^{-1}$. Dark histograms indicate the RX J1548.9+0851 system.

To determine radial velocities and group memberships of the RX J1548.9+0851 candidate faint galaxy population, the crosscorrelation technique as described by Tonry \& Davis (1979) was applied by means of the xcsao task in the IRAF rvsao package. The analysis yielded redshifts for 84 targets ( $\sim 93 \%$ of all extracted spectra). Stellar templates of zero radial velocity were taken from the library of stellar spectra by Jacoby et al. (1984). Spectral types similar to the stellar populations of ellipticals, i.e. $\mathrm{G}$ and $\mathrm{K}$ giants have been selected for that purpose. To quantify the reliability of the redshift measurements, the confidence parameter $R$ provided by xcsao has been considered. Kurtz \& Mink (1998) have calibrated $R$ empirically and state that the redshift determination is reliable above $R_{\min }=3.0$. The final adopted redshifts were estimated as the average from all utilised stellar templates whilst redshift errors were estimated from the associated standard deviations. Ultimately, redshifts were transformed to radial velocities by means of the relativistic Doppler effect.

\section{The RX J1548.9+0851 system}

Figure 1 shows the distribution of radial velocities for all 84 galaxies with measured redshifts in the observed VIMOS field. The RX J1548.9+0851 group is well-defined in radial-velocity space exhibiting a strong peak around $v_{r} \sim$ $21000 \mathrm{~km} \mathrm{~s}^{-1}$ (dark histograms), confirming that it constitutes a real gravitationally bound system. A galaxy with a radial velocity $v_{i}$ was considered a group member if the condition $\left|v_{i}-v_{\text {sys }}\right|<\Delta v$ is fulfilled. According to Ramella et al. (1994), a $\Delta v$ of $1500 \mathrm{~km} \mathrm{~s}^{-1}$ is a good match to the scale of loose groups and was used in this work. The median radial velocity of the dark histograms in Fig. $1\left(v_{\text {med }}=v_{\text {sys }}=20696 \mathrm{~km} \mathrm{~s}^{-1}\right)$ was considered as system velocity. The thus defined sample of group members holds until a $\Delta v$ of $1250 \mathrm{~km} \mathrm{~s}^{-1}$ is applied ${ }^{3}$.

Out of the 84 measured redshifts, 41 ( $49 \%)$ have been identified as group members in the whole VIMOS field, amounting to 40 identified members within $1 \mathrm{Mpc}$ of the central elliptical. Complementing the present measurements, 31 additional redshifts have been found in the literature, resulting in a total number of 54 spectroscopically confirmed group members within $1 \mathrm{Mpc}$, amounting to $\sim 51 \%$ of all studied redshifts within this radius (see also Fig. 3). Figure 2 shows the VIMOS $R$ band

\footnotetext{
3 Accounting also for radial velocity error bars.
}

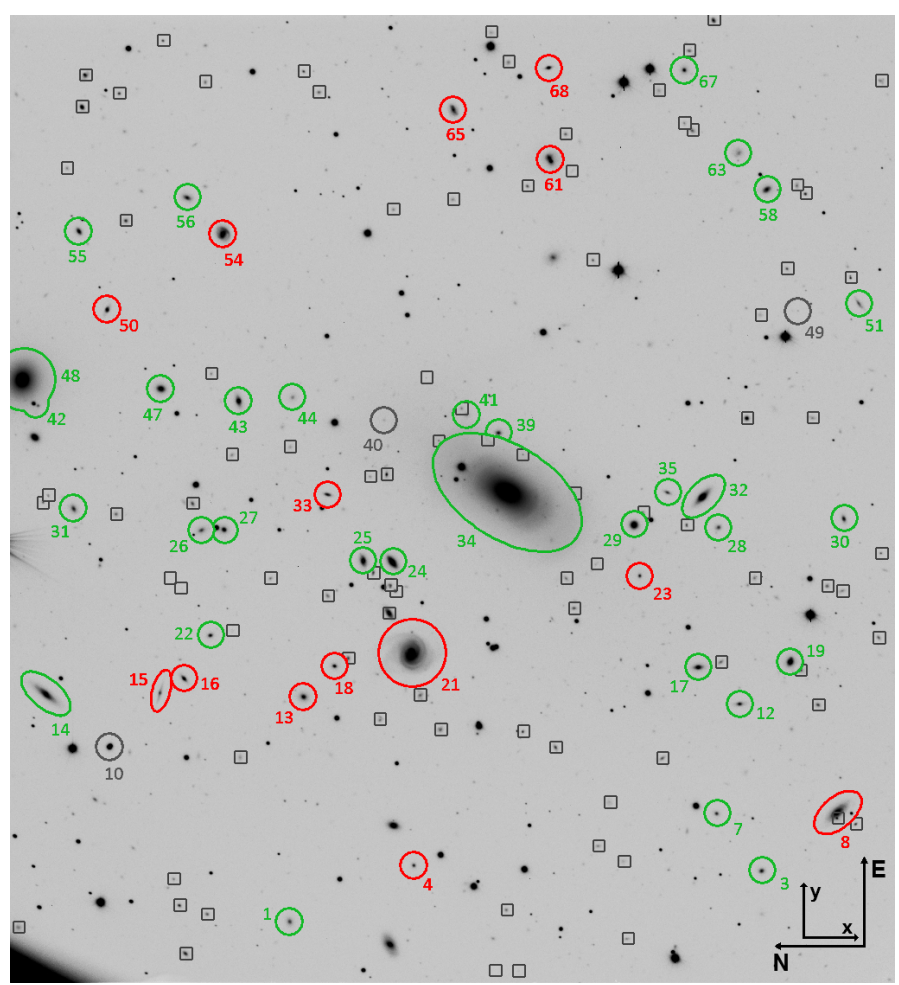

Fig. 2. Spectroscopic targets in the central region of the RX J1548.9+0851 system. Green circles indicate group members whilst red ones are background galaxies. Grey circles are spectroscopic targets without a reliable redshift. Squares show photometrically classified galaxies in the SDSS down to $i^{\prime}=21 \mathrm{mag}$. The field-of-view is $\sim 7 \times 8$ arcmin (VIMOS quadrant 3 - orientation following the CCD mask coordinates $(x, y)$. The alignment of equatorial coordinates $(N, E)$ is also shown). Numbers refer to the ID of Table 4.

pre-image of the inner region of the RX J1548.9+0851 system. Green circles indicate spectroscopically confirmed group members whilst red ones are background galaxies. Squares show photometrically classified galaxies in the SDSS down to $i^{\prime}=$ $21 \mathrm{mag}$. The inner region of the system is dominated by two bright ellipticals instead of only one as expected for a fossil group. The second brightest elliptical is located at a projected distance of $326 \mathrm{kpc}$ from the central galaxy. With a magnitude difference of $\Delta m_{1,2}=1.34$ in the $r^{\prime}$ band, it clearly violates the 2 mag criterion from Jones et al. (2003) leaving the question of RX J1548.9+0851 being a fossil or not to the definition of the virial radius.

Table 4 lists, amongst others, the radial velocities and associated confidence parameters $R$ of the studied group population within $1 \mathrm{Mpc}$ of the central elliptical. Galaxies are listed with increasing right ascension, i.e. from bottom to top in Fig. 2.

\subsection{The colour-magnitude diagram}

Figure 3 shows colour-magnitude diagrams of all SDSS galaxies down to $i^{\prime}=21 \mathrm{mag}$ within $300 \mathrm{kpc}$ and $1 \mathrm{Mpc}$ of the central elliptical. The magnitude limit of the spectroscopic target selection is indicated as vertical line. At a luminosity distance of $D_{\mathrm{L}}=326 \mathrm{Mpc}$ based on the median redshift of the system, this magnitude limit corresponds to an absolute magnitude of $M_{i^{\prime}} \sim-16.9$ mag. Objects with untypical magnitudes and colours lying outside the main point distribution have been checked in SDSS. Most of these objects were identified as stars misclassified as galaxies and were removed from the 


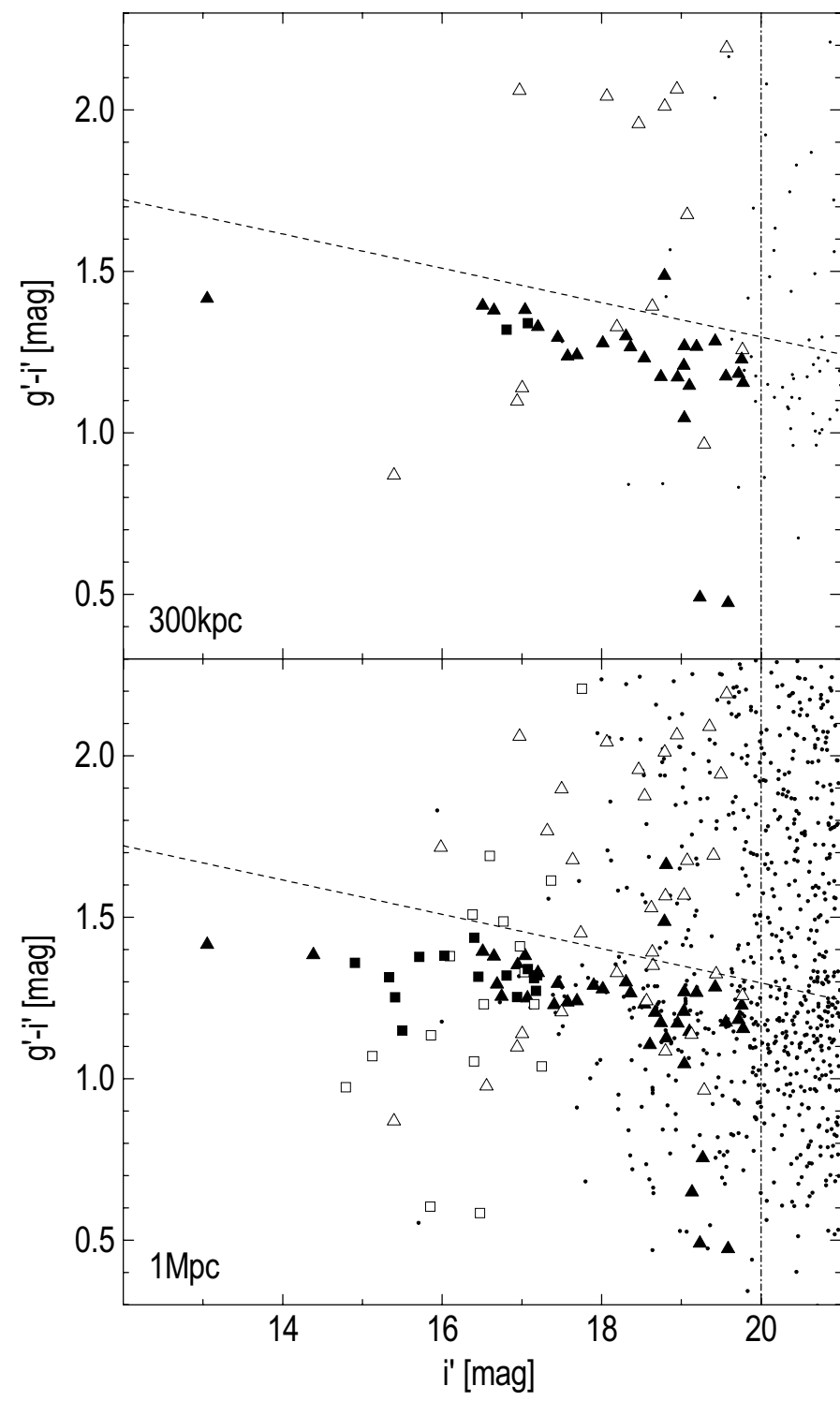

Fig. 3. Colour-magnitude diagrams for all galaxies within $300 \mathrm{kpc}$ and $1 \mathrm{Mpc}$. Galaxies with spectra are shown as triangles (this work) and squares (literature). Small dots are photometrically classified SDSS galaxies with $i^{\prime}<21$ mag. Filled symbols show spectroscopically confirmed group members whilst open ones are background galaxies. Group members form a tight red sequence of $\sim 0.4$ mag thickness. The dashed lines show the adopted upper limit of this sequence $g^{\prime}-i^{\prime}=-0.05317 \times i^{\prime}+2.36$. Vertical lines show the magnitude limit $\left(i^{\prime}=20 \mathrm{mag}\right)$ for the spectroscopic target selection.

plot. Small dots are all SDSS sources in the field brighter than $i=21 \mathrm{mag}$, photometrically classified as galaxies. Triangles show the measured redshifts from this work whilst squares indicate the additional redshifts found in SDSS and NED. Filled symbols show confirmed group members whilst open ones are background galaxies. The diagrams clearly reveal the increasing incompleteness of redshift measurements towards fainter magnitudes. The faintest galaxy with a reliable redshift in the investigated sample has a magnitude of $i^{\prime}=19.78 \mathrm{mag}$. Also the spatial incompleteness towards larger radii is evident. Whilst the spectroscopically studied sample within $300 \mathrm{kpc}$ is well represented above $i^{\prime}=20$ mag, having 68\% measured redshifts compared to all SDSS galaxies in the field, this fraction drops to $24 \%$ within $1 \mathrm{Mpc}$. Nearly all redshifts from the literature are
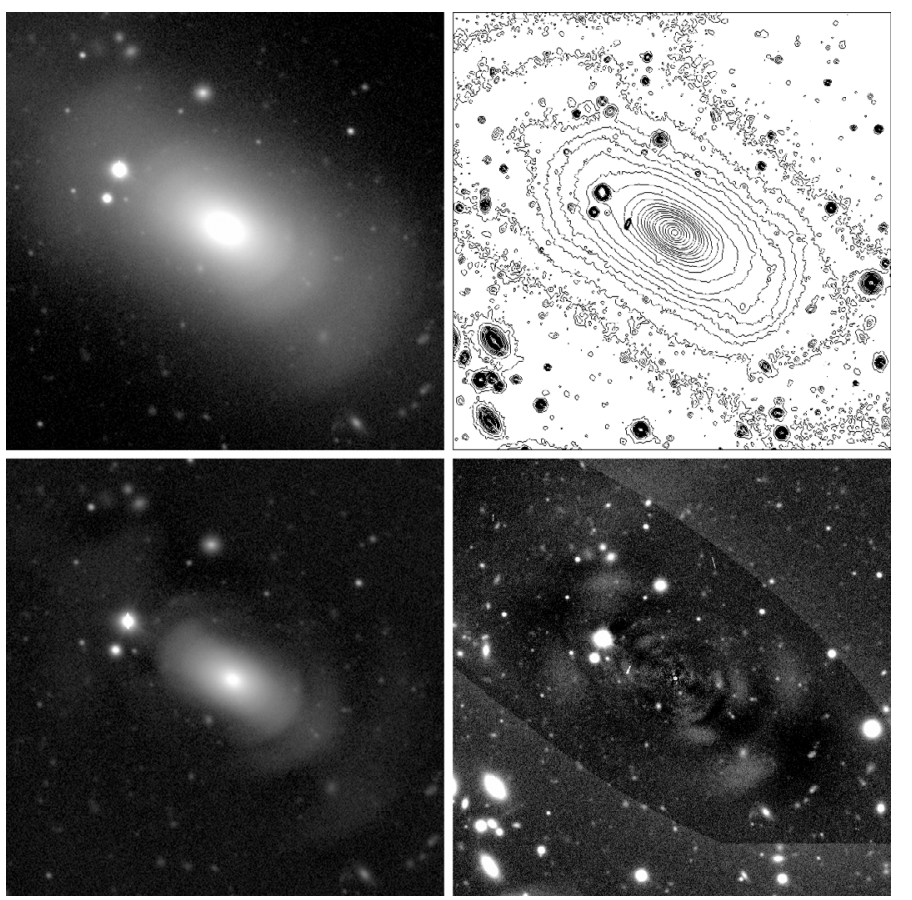

Fig. 4. Morphology of the central elliptical. Upper left: $R$ band image, upper right: isophote map, lower left: $R$ band image after subtraction of the median box filter, lower right: residuals to the IRAF ellipse model. Shells are clearly visible in the lower panels and show strong symmetry along the major axis. The isophote map reveals boxy isophotes in the outer regions of the galaxy, resulting from the observed shell structure. The residuals illustrate the large contamination of foreground sources which have been masked within the ellipse task. The field of view is $\sim 1.5 \operatorname{arcmin}$ on a side in the left panels and $\sim 2.2 \operatorname{arcmin}$ in the right ones.

found in the outer regions of the system, where no VIMOS measurements were taken. Group members form an unambiguous red sequence from the brightest galaxy into the dwarf regime whilst non-members are only diffusely distributed in the diagrams. The dashed lines in Fig. 3 indicate the adopted red sequence upper limit. Only 2 members are found above this upper limit. Interestingly, these 2 galaxies (39 and 42) are located in the immediate vicinity of the two brightest ellipticals in the group (see Fig. 2). The identified red sequence of spectroscopically confirmed group members is well-defined in colour and shows a lack of galaxies that are bluer than the adopted red sequence upper limit by $\geq 0.4 \mathrm{mag}$. Galaxies bluer than this limit are at least $\sim 6$ mag fainter than the central elliptical and comprise only 4 galaxies in the whole spectroscopic sample, confirming that the RX J1548.9+0851 system is dominated by old and passively evolving galaxies.

\subsection{The central elliptical}

Since the central elliptical in fossil groups is supposed to contain the merger history of the progenitor group population, its morphological appearance is of special interest. Figure 4a shows the central elliptical in the VIMOS $R$ band pre-image. Symmetric shells are unambiguosly identified along the galaxy major axis. To enhance the appearance of these features, the image was smoothed in IRAF by means of a median box filter and subtracted by this unsharp mask. The residuals reveal the observed shell structure in much more detail (Fig. 4c) and confirm the strong symmetry as already indicated by the unaltered frame. In 


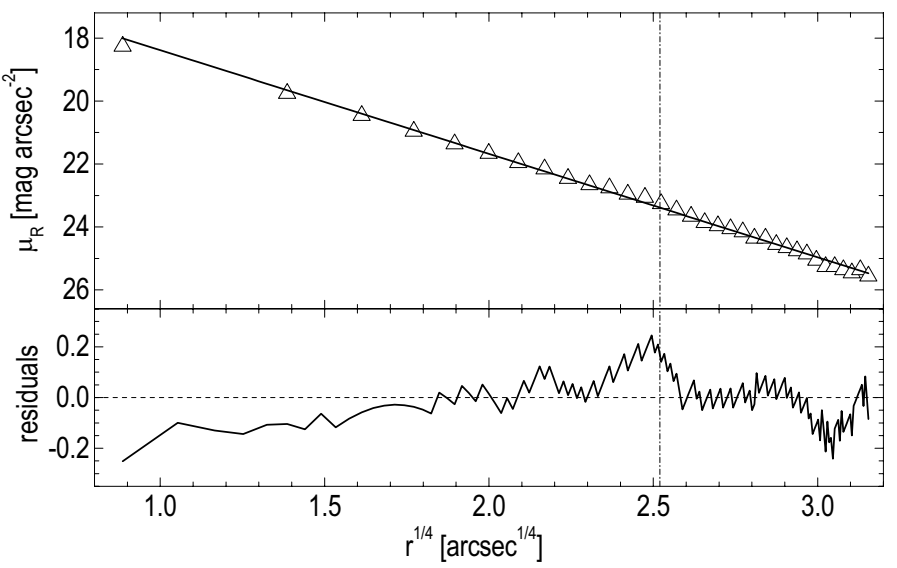

Fig. 5. Surface brightness profile of the central elliptical plotted against $r^{1 / 4}$. The straight line is the deVaucouleurs fit to the data. The vertical line indicates the effective radius. The lower panel shows the residuals to the fit. No light excess characteristic for a $\mathrm{cD}$ galaxy is seen.

addition, the surface brightness profile of the central elliptical has been studied with IRAF ellipse. Due to the galaxy's large spatial extent, its diffuse light distribution is strongly contaminated by numerous smaller objects. Therefore care was taken in masking out all these sources properly in the isophote fitting procedure to avoid systematic deviations in the resulting surface brightness profile. Ellipticity, position angle and central coordinates of the successive ellipses were allowed to change with radius. Figure 4d shows the residuals between the $R$ band image and the obtained isophote model, again revealing the pronounced shell structure. To quantify the shape of the surface brightness profile, a deVaucouleurs $r^{1 / 4}$ law was fitted to the data from outside the seeing dominated centre $(0.7 \mathrm{arcsec})$ down to $\mu_{R} \sim 25 \mathrm{mag} \operatorname{arcsec}^{-2}$. The effective radius was estimated to $r_{\mathrm{e}}=40 \operatorname{arcsec}$ corresponding to $\sim 55 \mathrm{kpc}$. Figure 5 shows the profile in $\mu_{R}$ mag $\operatorname{arcsec}^{-2}$ against $r^{1 / 4}$. The solid line is the bestfit deVaucouleurs law. Residuals indicate only minor deviations from the model in the order of $0.1 \mathrm{mag}$. No light excess above the deVaucouleurs law is seen that would classify the elliptical as a $\mathrm{cD}$ galaxy.

\subsection{SSP ages and metallicities of group members}

To determine ages and metallicities of the RX J1548.9+0851 group population, the open-source package ULySS ${ }^{4}$ (University of Lyon Spectroscopic Analysis Software; Koleva et al. 2009) was used to fit synthetic single stellar population (SSP) models based on a library of stars with varying atmospheric parameters directly to the observed galaxy spectra yielding SSP equivalent ages and metallicities of the stellar populations of the investigated galaxy sample. ULySS makes use of the Pegase HR models from Le Borgne et al. (2004) based on the Elodie 3.1 library of stellar spectra (Prugniel \& Soubiran 2001; Prugniel et al. 2007). Since the synthesis of a stellar population requires a stellar spectrum at any point in the parameter space $\left(T_{\text {eff }}, \log g\right.$ and $[\mathrm{Fe} / \mathrm{H}])$, the library involves an interpolator for that purpose. All Pegase HR models are computed assuming a Salpeter IMF and Padova 1994 evolutionary tracks providing synthetic SSPs with ages between 1-20000 Myr and metallicities between $-2.3-0.69$ dex. A multiplicative polynomial is used to adjust the overall spectral shape to the SSP model. The order of

\footnotetext{
${ }^{4}$ http://ulyss.univ-lyon $1 . f r /$
}
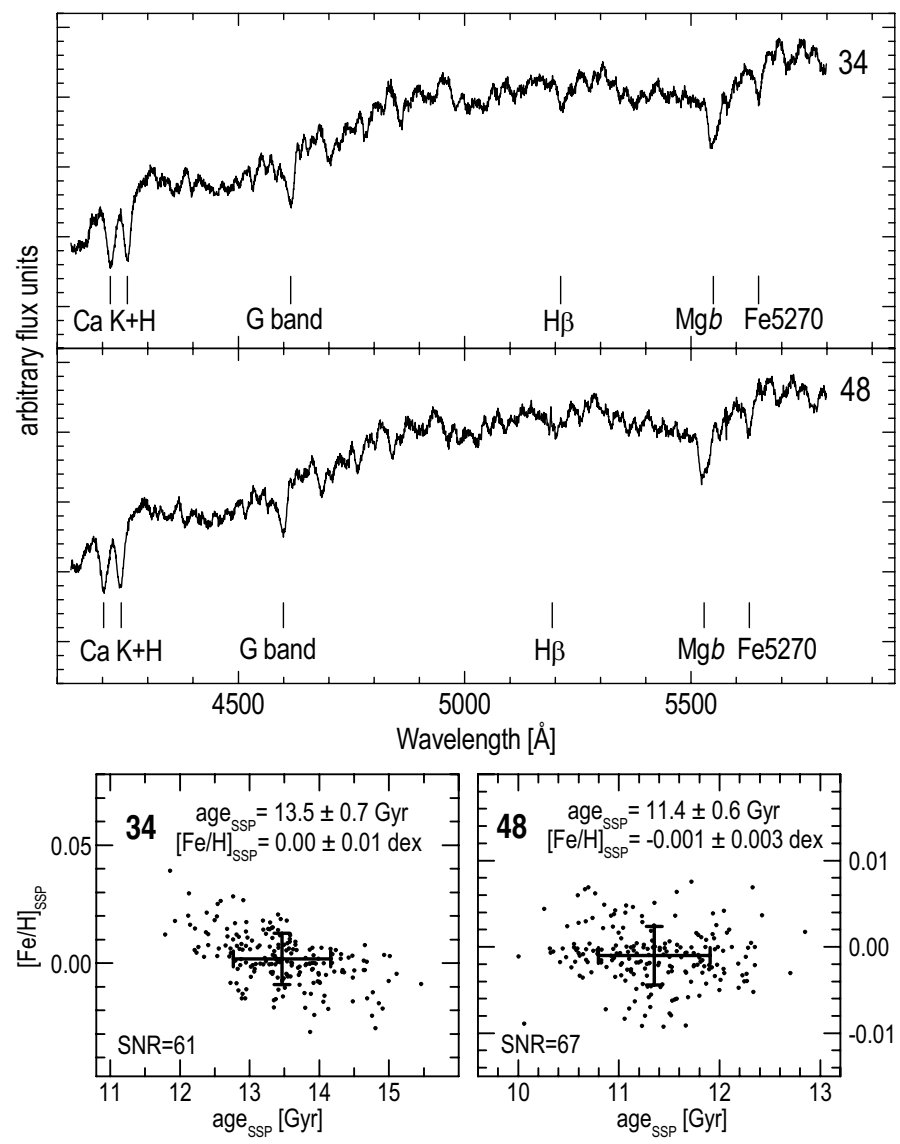

Fig. 6. Upper panels: spectra of the two brightest ellipticals in the RX J1548.9+0851 system. The most prominent absorption features are identified. As expected, the galaxies are dominated by an old stellar population. Lower panels: Monte-Carlo simulations of the corresponding SSP fits. Crosses indicate averages and standard deviations of each point distribution, presenting the final adopted fitting results.

the polynomial can be freely chosen by the user but shouldn't be taken too low resulting mostly in a severe mismatch of the investigated spectrum and the SSP model. Balancing between a minimum order to achieve reasonable, stable values and a maximum order to avoid long, unnecessary computing time, an order of 40 was chosen for all galaxies. To evaluate the reliability of the performed SSP fits, $\chi^{2}$ and convergence maps of the investigated age-metallicity parameter space have been considered. 200 Monte-Carlo simulations have been computed for every galaxy, repeating a fit successively with random Gaussian noise ${ }^{5}$ applied to the spectrum in each step. The resulting point distributions were then used to calculate average SSP ages and metallicities and the associated standard deviations (excluding obvious outliers). Figure 6 shows the spectra of the two brightest ellipticals in the RX J1548.9+0851 system and the resulting Monte-Carlo simulations of the corresponding SSP fits. Table 4 lists the measured SSP equivalent ages and metallicities of all RX J1548.9+0851 group members within $1 \mathrm{Mpc}$.

To investigate the correlation of the measured SSP ages with the spatial distribution of member galaxies, Fig. 7 shows ages of galaxies in the inner $\sim 300 \mathrm{kpc}$ region of the RX J1548.9+0851 system. Galaxies have been separated into old and young objects at an arbitrary age threshold of $8 \mathrm{Gyr}$. There is a clear segregation

5 The dimension of the added noise was based on a user-defined signalto-noise ratio provided for each galaxy. The $\mathrm{S} / \mathrm{N}$ ratios were measured with IRAF splot at a rest-frame wavelength of $\sim 5140 \AA$. 


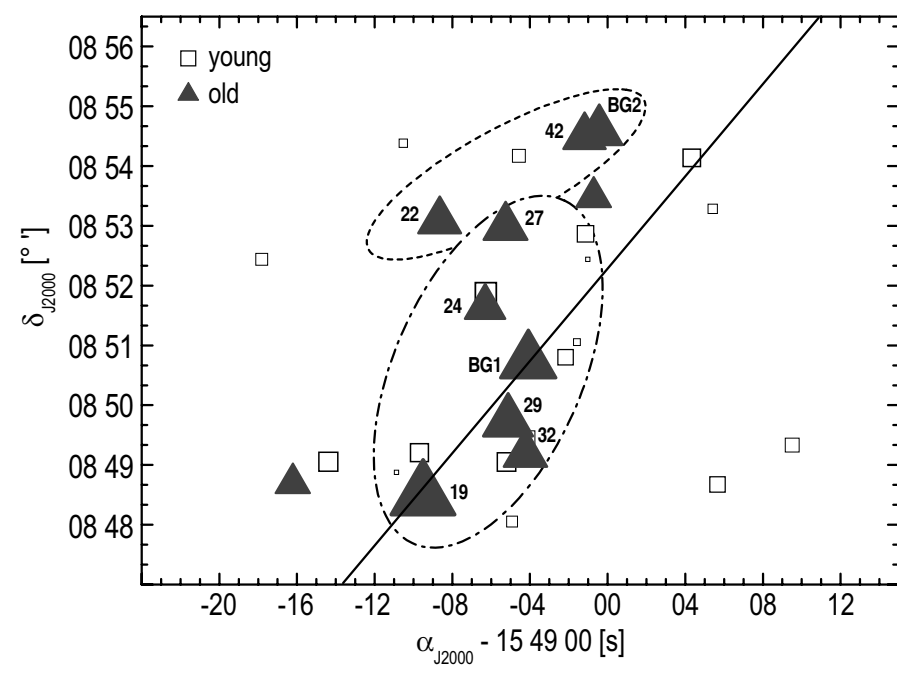

Fig. 7. Age map of the inner $\sim 300 \mathrm{kpc}$ region of RX J1548.9+0851 (see Fig. 2). There is a clear segregation in age for the investigated faint galaxy population. Whilst younger objects are diffusely distributed and found mostly in the outskirts of the system, the oldest objects (>8 Gyr) form a clear elongated structure aligned with the orientation of the major axis of the central elliptical, indicated as solid line. Ellipses contain the same objects as those in Fig. 8. See text for details.

in age for the investigated galaxy population. Whilst younger objects are diffusely distributed and found mostly in the outskirts of the system, the oldest objects form a clear elongated structure aligned with the orientation of the major axis of the central elliptical, indicated as dashed line.

\subsection{Galaxy velocity distribution}

Figure 8a shows the galaxy velocity distribution of spectroscopically confirmed group members in the inner $\sim 300 \mathrm{kpc}$ of the system $(N=32)$ whilst Fig. 8b relates the measured radial velocities with the corresponding SSP ages. Based on the spatial segregation in age found in Fig. 7, galaxies are again separated into old and young objects at the arbitrary age of 8 Gyr. The two brightest ellipticals, hereafter BG1 and BG2, are the oldest objects in the sample (with the exception of one faint galaxy, exhibiting a huge error bar in age, however) and build up the centres of two subsamples in the velocity distribution of old galaxies (cross-hatched histograms). Whilst the oldest galaxies around BG1 form the core of the overall velocity distribution, old galaxies around BG2 are found at systematically lower velocities. Hence, the luminosity-weighted mean radial velocity $v_{r}=20633 \mathrm{~km} \mathrm{~s}^{-1}$ is significantly lower than the radial velocity of the central elliptical $v_{r}=20854 \mathrm{~km} \mathrm{~s}^{-1}$. In contrast to the old galaxy population, young objects show a smooth distribution over all velocities and exhibit a much larger dispersion. Dashed ellipses in Figs. 7 and 8b contain the same objects, indicating that the subpopulations around BG1 and BG2 in radial velocity space are also more or less spatially correlated.

\subsection{Velocity dispersion}

To evaluate the dynamical properties of the RX J1548.9+0851 system, the luminosity weighted dynamical formulae as presented in Firth et al. (2006) have been applied. Table 1 lists the luminosity-weighted and non-weighted velocity dispersions for all spectroscopically confirmed group members within $1 \mathrm{Mpc}$ and for the sample of the inner region shown in Fig. 7 and 8.

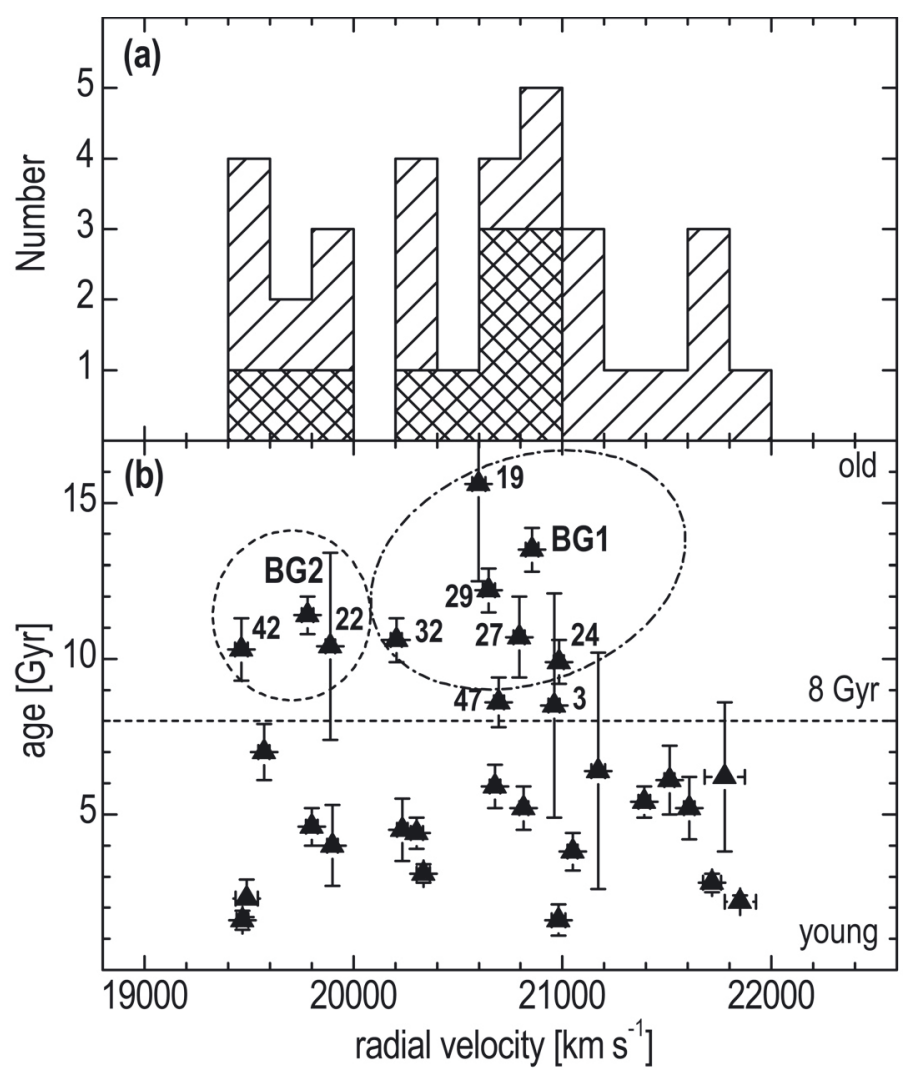

Fig. 8. Galaxy velocity distribution of the inner $\sim 300 \mathrm{kpc}$ region of the RX J1548.9+0851 system $(N=32)$ correlated with the measured SSP ages a) Galaxy velocity distribution. Old galaxies $(>8 \mathrm{Gyr})$ are indicated as cross-hatched histograms. b) Age-velocity plane. Galaxies are separated into old and young objects. Ellipses contain the same objects as those in Fig. 7 and separate old galaxies into subsamples around BG1 and BG2. See text for details.

Dispersions have also been calculated excluding young galaxies and BG2. Focusing on all spectroscopically confirmed members within $1 \mathrm{Mpc}$ leads to the largest velocity dispersion $\left(568 \mathrm{~km} \mathrm{~s}^{-1}\right)$ whilst restricting to the inner $\sim 300 \mathrm{kpc}$ region leads to a slightly smaller value $\left(496 \mathrm{~km} \mathrm{~s}^{-1}\right)$. The old population within $\sim 300 \mathrm{kpc}$ yields a value of $440 \mathrm{~km} \mathrm{~s}^{-1}$. This restriction does not lower the measured dispersion as severely as the exclusion of BG2 which dominates the luminosity of all other faint and young galaxies by far. Omitting BG2 leads to much smaller values in the central region for both the whole $\left(321 \mathrm{~km} \mathrm{~s}^{-1}\right)$ and old (266 $\mathrm{km} \mathrm{s}^{-1}$ ) galaxy populations. Non-weighted dispersions show significantly higher values because of the large spread in velocity of young, faint galaxies. The presented results are in agreement with the study of Mendes de Oliveira et al. (2006) who found that emission-line galaxies primarily populate the outskirts of the galaxy velocity distribution of the fossil cluster RX J1552.2+2013 suggesting that young, star-forming galaxies have recently been accreted by the group and are not yet virialised.

\subsection{Dynamical mass}

Table 2 summarises the luminosity-weighted dynamical properties of RX J1548.9+0851 derived from all spectroscopically confirmed group members within $1 \mathrm{Mpc}$. Weightings $w_{i}$ are based on the apparent SDSS $r^{\prime}$ band magnitudes of group member galaxies, converted to relative luminosities. The dynamical mass 
Table 1. Velocity dispersion measurements.

\begin{tabular}{lccccc}
\hline \hline & $(1)$ & $(2)$ & $(3)$ & $(4)$ & $(5)$ \\
\hline$N$ & 54 & 32 & 31 & 11 & 10 \\
$\sigma=\left[\frac{1}{N} \sum_{i} \Delta v_{i}^{2}\right]^{\frac{1}{2}}$ & 693 & 720 & 716 & 499 & 475 \\
$\sigma_{\text {lum }}=\left[\frac{\sum_{i} w_{i}\left(\Delta v_{i}\right)^{2}}{\sum_{i} w_{i}}\right]^{\frac{1}{2}}$ & 568 & 496 & 321 & 440 & 266 \\
\hline
\end{tabular}

Notes. All values in $\mathrm{km} \mathrm{s}^{-1}$. (1) Calculations for all spectroscopically confirmed members within $1 \mathrm{Mpc}$ including redshifts from literature $(N=54) .{ }^{(2)}$ Calculations for all members within $\simeq 300 \mathrm{kpc}-$ sample as shown in Figs. 7 and $8(N=32) .{ }^{(3)}$ Same as (2) excluding BG2 $(N=31) .{ }^{(4)}$ Restricting to old $(>8 \mathrm{Gyr})$ galaxies $(N=11) .{ }^{(5)}$ Same as (4) excluding BG2 $(N=10)$.

of RX J1548.9+0851 was determined using two different mass estimators, i.e. virial and projected mass. For that purpose, the harmonic mean and virial radii have been calculated. The computation of the harmonic mean radius $R_{\mathrm{H}}$ (see Fig. 9) involves the determination of the distances $\left|r_{i}-r_{j}\right|$ of all $N(N-1) / 2=$ 1431 galaxy pairs within the sample. For the projected mass, the projected radial distances $r_{\perp i}$ from the central elliptical have been applied. Characteristic system crossing times were estimated by dividing the average projected distance of group members from the central elliptical by the average speed of group members relative to the group centre of mass, considered as the central elliptical. Finally, the mean mass from both estimates is given with the corresponding standard deviation as error estimate. As already expected by the relatively large velocity dispersions as shown in Table 1 the system shows a comparatively large dynamical mass, typical for a cluster rather than a group, in agreement with previous work (Mendes de Oliveira et al. 2006, 2009; Cypriano et al. 2006; Proctor et al. 2011). The crossing times below 1 Gyr for the inner $300 \mathrm{kpc}$ of RX J1548.9+0851 suggest that members had enough time to virialise within the group potential.

\subsection{The galaxy luminosity function}

Following previous work on fossils, the shape of the galaxy luminosity function of RX J1548.9+0851 has been studied in the SDSS $g^{\prime}, r^{\prime}$, and $i^{\prime}$ bands. To correct for incompleteness in the sample of spectroscopically determined group members, photometric redshift models have been adopted from the SDSS $^{6}$ for all galaxies with no observed spectrum. These additional photometric redshifts confirmed the clear segregation of galaxies into members and non-members at the red sequence upper limit. Figure 10 shows the galaxy luminosity distribution of RX J1548.9+0851 member galaxies in the SDSS $g^{\prime}, r^{\prime}$, and $i^{\prime}$ bands within $1 \mathrm{Mpc}$ of the central elliptical. Open histograms show all SDSS galaxies whilst cross-hatched ones refer to spectroscopically confirmed members. Dashed histograms indicate photometrically determined group members and evidently dominate the studied group population at fainter magnitudes. Solid lines show the corresponding best-fit Schechter functions. The

\footnotetext{
${ }^{6}$ Three photometric redshift models are stored in the SDSS. To evaluate the accuracy of these models, the derived VIMOS redshifts have been compared with the corresponding photometric redshift data from the SDSS. Based on this comparison, redshift thresholds for group membership have been defined in all three models. A galaxy was considered a photometric group member only if it was considered as such in all three models.
}

Table 2. Luminosity weighted dynamical properties of the RX J1548.9+0851 system.

\begin{tabular}{lcc}
\hline \hline$(1)$ & $(2)$ & $(3)$ \\
\hline Mean radial velocity & $\bar{v}=\frac{\sum_{i} w_{i} v_{i}}{\sum_{i} w_{i}}$ & $20731 \mathrm{~km} \mathrm{~s}^{-1}$ \\
Velocity dispersion & $\sigma_{\mathrm{lum}}=\left[\frac{\sum_{i} w_{i}\left(\Delta v_{i}\right)^{2}}{\sum_{i} w_{i}}\right]^{\frac{1}{2}}$ & $568 \mathrm{~km} \mathrm{~s}^{-1}$ \\
Harmonic radius & $R_{\mathrm{H}}=\left[\frac{\sum_{i} \sum_{j<i}\left(w_{i} w_{j}\right) /\left(r_{i}-r_{j}\right)}{\sum_{i} \sum_{j<i}\left(w_{i} w_{j}\right)}\right]^{-1}$ & $388 \mathrm{kpc}$ \\
Virial radius & $r_{\mathrm{V}}=\frac{\pi R_{\mathrm{H}}}{2}$ & $609 \mathrm{kpc}$ \\
Crossing time & $t_{\mathrm{c}}=\frac{\langle r\rangle}{\langle v\rangle}$ & $0.68 \mathrm{Gyr}$ \\
Virial mass & $M_{\mathrm{V}}=\frac{3}{G} \sigma^{2} r_{\mathrm{V}}$ & $1.37 \times 10^{14} M_{\odot}$ \\
Projected mass & $M_{\mathrm{p}}=\frac{32}{\pi G} \frac{\sum_{i} w_{i}\left(\Delta v_{i}\right)^{2} r_{\perp i}}{\sum_{i} w_{i}}$ & $3.70 \times 10^{14} M_{\odot}$ \\
Mean mass & $1 / 2\left(M_{\mathrm{V}}+M_{\mathrm{p}}\right)$ & $2.5 \pm 0.8 \times 10^{14} M_{\odot}$ \\
\hline
\end{tabular}

Notes. Velocities are given in $\mathrm{km} \mathrm{s}^{-1}$, radii in kpc, masses in $10^{14} M_{\odot}$, and the crossing time in Gyr. ${ }^{(1)}$ Description. (2) Dynamical formulae. ${ }^{(3)}$ Calculations for all spectroscopically confirmed members within $1 \mathrm{Mpc}$ including redshifts from literature $(N=54)$.

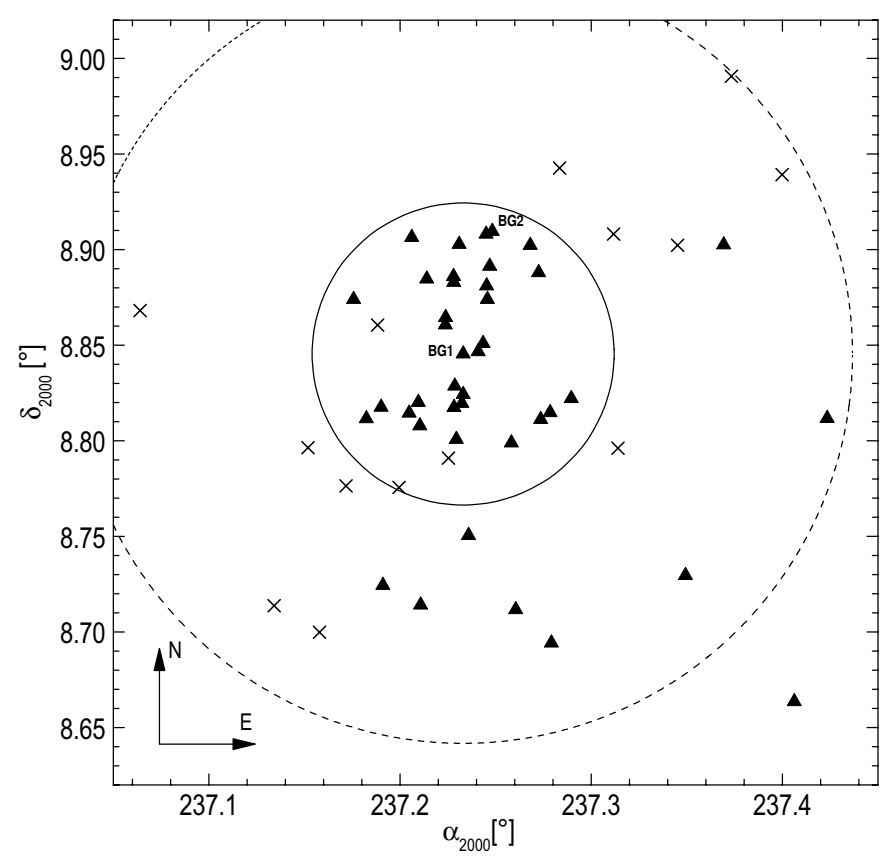

Fig. 9. Spatial distribution of spectroscopically confirmed group members from this work (triangles) and the literature (crosses). The inner circle shows the luminosity-weighted harmonic mean radius centred on the dominating elliptical. The dashed circle indicates a radius of $1 \mathrm{Mpc}$. The two brightest ellipticals are marked as BG1 and BG2.

central elliptical was excluded in this procedure. In all three passbands, the derived luminosity functions exhibit a steep faint end $\left(\alpha_{g^{\prime}}=-1.55, \alpha_{r^{\prime}}=-1.28, \alpha_{i^{\prime}}=-1.44\right)$ resulting in an average slope of $\alpha=-1.4 \pm 0.1$. The overall shape of the luminosity function doesn't reveal any peculiarities. No pronounced dip is visible at $M_{i^{\prime}}=-18+5 \log h$ as has been observed in the fossil RX J1552.2+2013 (Mendes de Oliveira et al. 2006).

\subsection{Mass-to-light ratio}

Total group luminosities have been estimated by adding up the luminosities of spectroscopically confirmed group members and galaxies photometrically classified as members. In addition, group luminosities have also been determined by integrating 


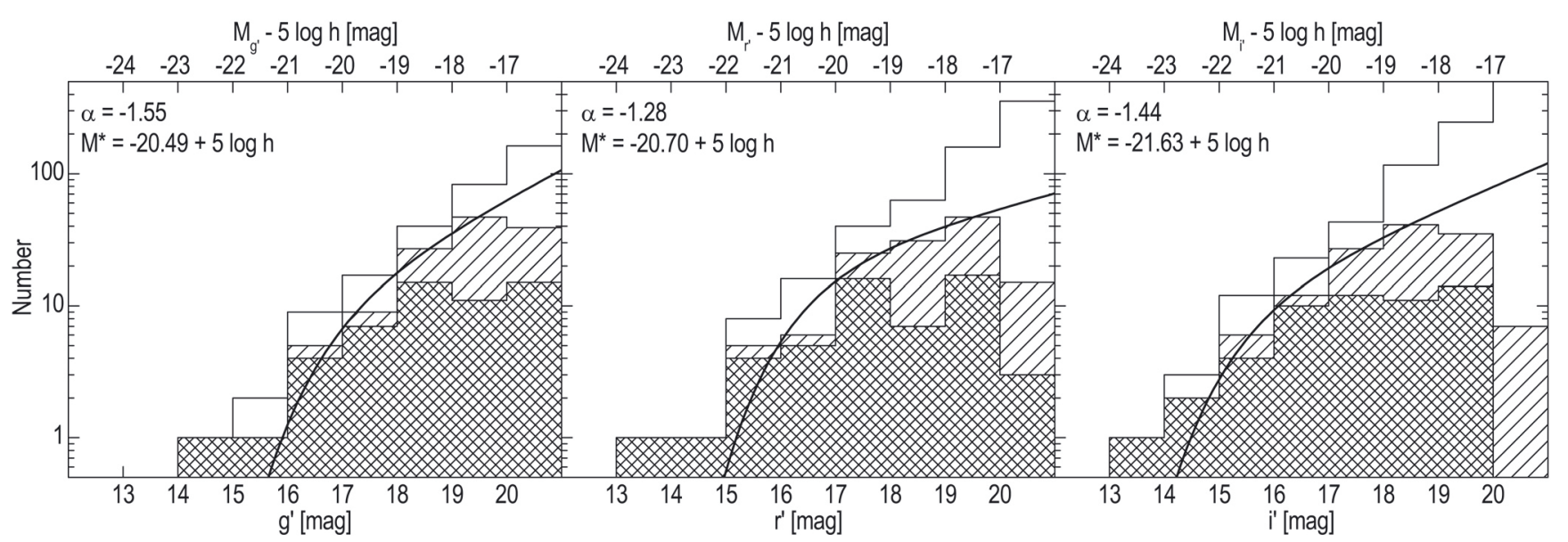

Fig. 10. The galaxy luminosity function of the RX J1548.9+0851 system in the SDSS $g^{\prime}, r^{\prime}$, and $i^{\prime}$ bands. Open histograms indicate all SDSS galaxies within $1 \mathrm{Mpc}$ whilst hatched ones show photometric members (see text) and cross-hatched ones the spectroscopically confirmed group sample. Solid lines are the best-fit Schechter functions. The central elliptical has been excluded in the fits. The Schechter fit parameters $\alpha$ and $M^{*}$ are shown for each passband.

Table 3. Group luminosities, luminosity function and mass-to-light ratios.

\begin{tabular}{lcccc}
\hline \hline & & $g^{\prime}$ & $r^{\prime}$ & $i^{\prime}$ \\
\hline$(1)$ & $L_{\mathrm{tot}}=\sum_{i} L_{i}$ & 5.22 & 6.35 & 7.62 \\
(2) & $L_{\mathrm{tot}}=\int_{0}^{\infty} L \Phi(L) d L$ & 6.25 & 5.90 & 8.41 \\
$(3)$ & $\alpha$ & -1.55 & -1.28 & -1.44 \\
$(4)$ & $M^{*}-5 \log h$ & -20.49 & -20.70 & -21.63 \\
$(5)$ & $M / L\left[M_{\odot} / L_{\odot}\right]$ & 479 & 394 & 328 \\
(6) & $L_{\mathrm{BG} 1} / L_{\mathrm{tot}}$ & $0.26(0.36)$ & $0.29(0.37)$ & $0.30(0.38)$ \\
\hline
\end{tabular}

Notes. Luminosities are given in $10^{11} L_{\odot}{ }^{(1)}$ Total luminosity of all member galaxies (hatched histograms). (2) Total luminosity derived from the best-fit Schechter function. ${ }^{(3,4)}$ Schechter function parameters. ${ }^{(5)}$ Mass-to-light ratios are calculated with the mean group mass and the luminosities from (1). ${ }^{(6)}$ Luminosity fraction of the central elliptical compared to the overall group luminosity. Numbers in parantheses refer to spectroscopically confirmed members only.

the best-fit Schechter function over all luminosity bins. The luminosity of the central elliptical was added to these latter values since the galaxy was previously excluded for the fit. Luminosities have been derived assuming that the absolute magnitude of the sun is $M_{g^{\prime} \odot}=5.14, M_{r^{\prime} \odot}=4.65$, and $M_{i^{\prime} \odot}=4.54^{7}$. Galaxy magnitudes were corrected for Galactic extinction $\left(A_{g^{\prime}}=\right.$ $0.15, A_{r^{\prime}}=0.11$, and $\left.A_{i^{\prime}}=0.08\right)$ and $k$-correction $\left(k_{g^{\prime}}=0.22\right.$, $k_{r^{\prime}}=0.09$, and $\left.k_{i^{\prime}}=0.04\right)$ before converting them to luminosities $^{8}$. The luminosity fraction of the central elliptical compared to the overall group luminosity budget amounts to $\sim 30 \%$ which is significantly lower than what has been observed for the fossil RX J1340.6+4018 ( 80\%; Mendes de Oliveira et al. 2009), most likely due to the presence of BG2 and the steep luminosity function. To estimate the mass-to-light ratio of the system, the mean group mass listed in Table 2 was considered. All luminosity estimates and the resulting mass-to-light ratios in the SDSS $g^{\prime}, r^{\prime}$, and $i^{\prime}$ bands are shown in Table 3 . The luminosity fraction of the central elliptical compared to the overall group luminosity budget is also presented. The resulting mass-to-light ratios are

\footnotetext{
7 Calculated by C. Willmer:

http://mips.as.arizona.edu/ cnaw/sun.html

8 Correction values are averages determined from the corresponding SDSS entries of all spectroscopically confirmed group members.
}

comparatively high showing values around $M / L \sim 400 M_{\odot} / L_{\odot}$ similar to the findings from other fossils (Vikhlinin et al. 1999; Khosroshahi et al. 2007) ${ }^{9}$.

\subsection{Scaling relations}

Figure 11 shows scaling relations between galaxy brightness, colour, line-of-sight velocity dispersion, SSP age and metallicity for the investigated RX J1548.9+0851 galaxy population. Velocity dispersions have been derived with the spectrum-fitting package ULySS, providing the amount of broadening applied to an SSP model to match a galaxy spectrum. Physically meaningful dispersions have been estimated by correcting this broadening for the instrumental dispersion of the spectrograph and the dispersion of the SSP model. Panels a)-d) relate the measured galaxy velocity dispersions with the remaining parameters. The diagrams show that galaxies tend to be brighter, redder, older, and more metal rich with increasing velocity dispersion. Panels e) and f) indicate a slight increase of galaxy brightness and colour with metallicity. No trend is observed when correlating SSP ages with SSP metallicities. Panels h) and i) show a clear trend between brightness, colour, and age. Especially colour shows a comparatively tight relation with age, only members 39 and 42 in the vicinity of the two brightest ellipticals being systematically redder. Panel j) shows the system's red sequence, the dash-dotted line presenting the adopted red sequence upper limit as seen in Fig. 3. The presented scaling relations clearly highlight the fact that more massive galaxies (higher velocity dispersions), are redder, brighter, older and more metal rich compared to less massive ones, implying also the observed relations without velocity dispersion (mass). This is plausible since more-massive galaxies, i.e. ellipticals, exhibit higher luminosities when assuming a constant mass-to-light ratio, can retain more metals due to their deeper potential wells, and are passively evolving with a dominating old stellar population absent of any young stars dominating the galaxy light in the blue.

9 The derived $r^{\prime}$ band $M / L$ ratio was converted to a corresponding value in $B$ to compare the result with the $M / L$ ratios from Khosroshahi et al. (2007). Uniform colours of $(B-R)=1.5$ (assuming all satellites to be early-types) and $(B-R)=0.8$ (assuming all satellites to be latetypes) were adopted, yielding upper and lower limits in the resulting $B$-band $M / L$ ratio of $200<M / L_{B}\left[M_{\odot} / L_{\odot}\right]<600$. 
Table 4. Identified group members in the observed VIMOS field within $1 \mathrm{Mpc}$ of the central elliptical.

\begin{tabular}{|c|c|c|c|c|c|c|c|c|c|}
\hline Galaxy $^{a}$ & $\alpha_{\mathrm{J} 2000^{b}}{ }^{b}$ & $\delta_{\mathrm{J} 2000^{b}}$ & $\begin{array}{c}i^{\prime c} \\
{[\mathrm{mag}]}\end{array}$ & $\begin{array}{l}g^{\prime}-i^{\prime c} \\
{[\mathrm{mag}]}\end{array}$ & $\begin{array}{c}v_{\mathrm{rad}}^{d} \\
{\left[\mathrm{~km} \mathrm{~s}^{-1}\right]}\end{array}$ & $R^{d}$ & $\begin{array}{c}\text { age }_{\text {SSP }}{ }^{e} \\
{[\mathrm{Gyr}]}\end{array}$ & $\begin{array}{c}{[\mathrm{Fe} / \mathrm{H}]_{\mathrm{SSP}}{ }^{e}} \\
\operatorname{dex}\end{array}$ & $\begin{array}{c}\sigma^{e} \\
{\left[\mathrm{~km} \mathrm{~s}^{-1}\right]}\end{array}$ \\
\hline$\overline{01 .}$. & 154842.24 & 085226.3 & 18.81 & 1.13 & $19899 \pm 26$ & 5.70 & $4.0 \pm 1.3$ & $-0.27 \pm 0.10$ & 14 \\
\hline 03. & 154843.86 & 084842.5 & 19.18 & 1.27 & $20960 \pm 27$ & 4.25 & $8.5 \pm 3.6$ & $-0.32 \pm 0.10$ & 46 \\
\hline 07. & 154845.70 & 084903.8 & 19.75 & 1.23 & $21172 \pm 33$ & 4.28 & $6.4 \pm 3.8$ & $-0.32 \pm 0.14$ & 43 \\
\hline 09 . & 154845.99 & 084324.6 & 16.94 & 1.36 & $21183 \pm 27$ & 5.64 & $3.6 \pm 0.7$ & $-0.07 \pm 0.05$ & 108 \\
\hline 12. & 154849.20 & 084853.0 & 19.03 & 1.05 & $19469 \pm 28$ & 4.04 & $1.6 \pm 0.3$ & $-0.46 \pm 0.10$ & 27 \\
\hline 14 . & 154849.51 & 085421.9 & 16.75 & 1.26 & $21716 \pm 42$ & 5.65 & $2.8 \pm 0.3$ & $0.07 \pm 0.06$ & 90 \\
\hline 17. & 154850.37 & 084912.6 & 18.00 & 1.28 & $21512 \pm 23$ & 5.36 & $6.1 \pm 1.1$ & $-0.15 \pm 0.05$ & 56 \\
\hline 19. & 154850.55 & 084828.9 & 17.19 & 1.33 & $20599 \pm 32$ & 5.73 & $15.6 \pm 3.1$ & $-0.29 \pm 0.03$ & 208 \\
\hline 20 . & 154850.73 & 084248.4 & 17.89 & 1.30 & $20632 \pm 36$ & 5.72 & $4.7 \pm 1.5$ & $-0.26 \pm 0.07$ & 47 \\
\hline 22 . & 154851.39 & 085304.0 & 19.02 & 1.21 & $19889 \pm 23$ & 5.05 & $10.4 \pm 3.0$ & $-0.62 \pm 0.06$ & 43 \\
\hline$\ldots$ & 154853.74 & 085137.7 & 16.64 & 1.38 & $20983 \pm 24$ & 9.34 & $9.9 \pm 0.7$ & $-0.15 \pm 0.03$ & 169 \\
\hline 25 . & 154853.78 & 085151.5 & 17.57 & 1.24 & $19572 \pm 26$ & 10.07 & $7.0 \pm 0.9$ & $-0.15 \pm 0.04$ & 94 \\
\hline $26 \ldots$ & 154854.75 & 085308.2 & 19.09 & 1.14 & $21851 \pm 74$ & 6.65 & $2.2 \pm 0.2$ & $0.05 \pm 0.05$ & 46 \\
\hline 27 & 154854.77 & 085257.5 & 18.35 & 1.26 & $20793 \pm 26$ & 10.65 & $10.7 \pm 1.3$ & $-0.19 \pm 0.04$ & 83 \\
\hline 28. & 154854.85 & 084902.9 & 19.55 & 1.18 & $21776 \pm 96$ & 6.30 & $6.2 \pm 2.4$ & $-0.40 \pm 0.11$ & 46 \\
\hline 29. & 154854.93 & 084943.0 & 17.03 & 1.39 & $20648 \pm 30$ & 10.11 & $12.2 \pm 0.7$ & $-0.17 \pm 0.02$ & 166 \\
\hline 30 . & 154855.12 & 084803.5 & 18.94 & 1.18 & $21049 \pm 24$ & 8.61 & $3.8 \pm 0.6$ & $-0.17 \pm 0.05$ & 49 \\
\hline 31. & 154855.44 & 085408.9 & 19.04 & 1.28 & $20303 \pm 29$ & 7.99 & $4.4 \pm 0.5$ & $-0.31 \pm 0.04$ & 56 \\
\hline$\ldots \ldots$ & 154855.82 & 084910.6 & 16.50 & 1.40 & $20205 \pm 27$ & 11.39 & $10.6 \pm 0.7$ & $-0.08 \pm 0.03$ & 182 \\
\hline $34 \ldots$ BG1 . & 154855.96 & 085042.9 & 13.05 & 1.42 & $20854 \pm 31$ & 10.15 & $13.5 \pm 0.7$ & $0.00 \pm 0.01$ & 309 \\
\hline 35 . & 154855.97 & 084927.2 & 19.71 & 1.19 & $20234 \pm 26$ & 7.84 & $4.5 \pm 1.0$ & $-0.36 \pm 0.07$ & 27 \\
\hline 36 . & 154856.71 & 084458.3 & 16.68 & 1.30 & $20558 \pm 26$ & 10.39 & $7.2 \pm 0.9$ & $-0.09 \pm 0.04$ & 119 \\
\hline 39 . & 154857.87 & 085047.3 & 18.78 & 1.49 & $20815 \pm 22$ & 10.87 & $5.2 \pm 0.7$ & $-0.16 \pm 0.04$ & 52 \\
\hline 41 . & 154858.45 & 085102.4 & 19.42 & 1.29 & $19489 \pm 53$ & 4.90 & $2.3 \pm 0.6$ & $-0.10 \pm 0.15$ & 85 \\
\hline 42 . & 154858.80 & 085427.9 & 18.81 & 1.67 & $19464 \pm 30$ & 12.90 & $10.3 \pm 1.0$ & $-0.07 \pm 0.03$ & 118 \\
\hline 43. & 154858.88 & 085250.6 & 17.68 & 1.24 & $21392 \pm 28$ & 11.88 & $5.4 \pm 0.5$ & $-0.05 \pm 0.03$ & 94 \\
\hline $44 \ldots$ & 154859.00 & 085225.0 & 19.77 & 1.15 & $20981 \pm 32$ & 5.02 & $1.6 \pm 0.5$ & $-0.17 \pm 0.22$ & 48 \\
\hline 47. & 154859.28 & 085327.5 & 17.46 & 1.30 & $20696 \pm 23$ & 12.90 & $8.6 \pm 0.8$ & $-0.14 \pm 0.03$ & 101 \\
\hline $48 \ldots \mathrm{BG} 2$. & 154859.56 & 085433.2 & 14.38 & 1.39 & $19781 \pm 28$ & 10.14 & $11.4 \pm 0.6$ & $-0.001 \pm 0.003$ & 321 \\
\hline 51. & 154902.01 & 084756.5 & 19.57 & 0.48 & $19606 \pm 34$ & 3.20 & - & - & - \\
\hline 52 & 154902.63 & 084240.0 & 19.26 & 0.77 & $20783 \pm 42$ & 2.92 & $3.9 \pm 3.0$ & $-0.25 \pm 0.34$ & - \\
\hline 55 . & 154904.31 & 085406.4 & 18.67 & 1.21 & $20678 \pm 25$ & 9.90 & $5.9 \pm 0.7$ & $-0.29 \pm 0.04$ & 64 \\
\hline 56. & 154905.38 & 085315.1 & 18.54 & 1.24 & $20334 \pm 31$ & 10.55 & $3.1 \pm 0.3$ & $0.14 \pm 0.05$ & 42 \\
\hline $58 \ldots \ldots$ & 154905.65 & 084840.1 & 18.29 & 1.31 & $21607 \pm 26$ & 11.06 & $5.2 \pm 1.0$ & $-0.14 \pm 0.06$ & 49 \\
\hline 63. & 154906.82 & 084853.2 & 19.22 & 0.50 & $21043 \pm 37$ & 3.24 & - & - & - \\
\hline $64 \ldots$ & 154907.05 & 084137.7 & 19.13 & 0.66 & $20225 \pm 37$ & 2.05 & $0.7 \pm 0.1$ & $-0.59 \pm 0.12$ & - \\
\hline $67 \ldots \ldots$ & 154909.47 & 084919.2 & 18.73 & 1.18 & $19801 \pm 28$ & 10.35 & $4.6 \pm 0.6$ & $-0.27 \pm 0.05$ & 53 \\
\hline $71 \ldots \ldots$ & 154924.05 & 084344.7 & 17.06 & 1.26 & $21512 \pm 39$ & 3.19 & $0.8 \pm 0.2$ & $-0.31 \pm 0.15$ & 14 \\
\hline $76 \ldots \ldots$ & 154928.82 & 085406.0 & 17.41 & 1.23 & $20756 \pm 29$ & 3.17 & $5.7 \pm 1.6$ & $-0.59 \pm 0.08$ & 42 \\
\hline $83 \ldots \ldots$ & 154941.70 & 084842.1 & 18.60 & 1.11 & $19873 \pm 24$ & 8.71 & $2.36 \pm 0.05$ & $-0.13 \pm 0.04$ & 62 \\
\hline
\end{tabular}

Notes. ${ }^{(a)}$ Galaxy ID referring to Fig. 2. ${ }^{(b)}$ Right ascension and declination shown in $h m s .{ }^{(c)}$ Model magnitudes from SDSS DR5. ${ }^{(d)}$ Radial velocities and corresponding confidence parameters $R$ determined with the IRAF xcsao package. ${ }^{(e)}$ SSP ages, metallicites and velocity dispersions determined with the software package ULySS.

\section{Discussion}

Using VIMOS multi-object spectroscopy, the galaxy population of the fossil candidate RX J1548.9+0851 has been studied in this work. The system was selected from the sample of fossil candidates presented in Santos et al. (2007). At a redshift of $z=0.0721$, the system lies at a luminosity distance of $D_{\mathrm{L}}=$ $326 \mathrm{Mpc}$ and an angular diameter distance of $D_{\mathrm{A}}=283 \mathrm{Mpc}$ corresponding to a spatial scale of $1.374 \mathrm{kpc} \operatorname{arcsec}^{-1}$. At this redshift the age of the universe amounts to $\sim 12.5 \mathrm{Gyr}$.

The optical appearance of RX J1548.9+0851 is dominated by two giant elliptical galaxies in the central region of the system, exhibiting a magnitude difference of $\Delta m_{1,2}=1.34$ in the SDSS $r^{\prime}$ band and a spectroscopic redshift difference of $\Delta z \sim 0.004$ (SDSS DR5). Although this magnitude difference at a projected distance of $326 \mathrm{kpc}$ clearly violates the 2 mag criterion for fossils, the system is listed in the sample of fossil candidates by Santos et al. (2007) who use a constant value of
$0.5 h_{70}^{-1} \mathrm{Mpc}$ to estimate half the group virial radius. The classification of RX J1548.9+0851 as fossil in Santos et al. (2007) is plausible though since these authors consider all satellites with $\Delta z>0.002$ as nonmember galaxies. Following the distribution of galaxies in radial velocity space as seen in Fig. 1, and considering the applied membership criterion of Ramella et al. (1994) $(\Delta z<0.005)$, there is no reason to classify the secondbrightest elliptical as background object in this work, however. At the group redshift of $z=0.0721$, the Evrard et al. (1996) estimate for half the virial radius yields a projected distance of at least $331 \mathrm{kpc}^{10}$ which would classify RX J1548.9+0851 as nonfossil. Estimating the virial radius via the luminosity-weighted

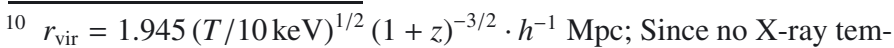
perature was derived for RX J1548.9+0851, a lower limit of $0.7 \mathrm{keV}$ was assumed. This value is based on the gas temperature - bolometric X-ray luminosity relation from the Millennium gas simulation (see Fig. 1 in Dariush et al. 2007) showing that a value of $0.7 \mathrm{keV}$ corresponds to about $L_{\mathrm{X} \text {,bol }}=10^{42} h^{-2} \mathrm{erg} \mathrm{s}^{-1}$, the X-ray limit of fossils as 


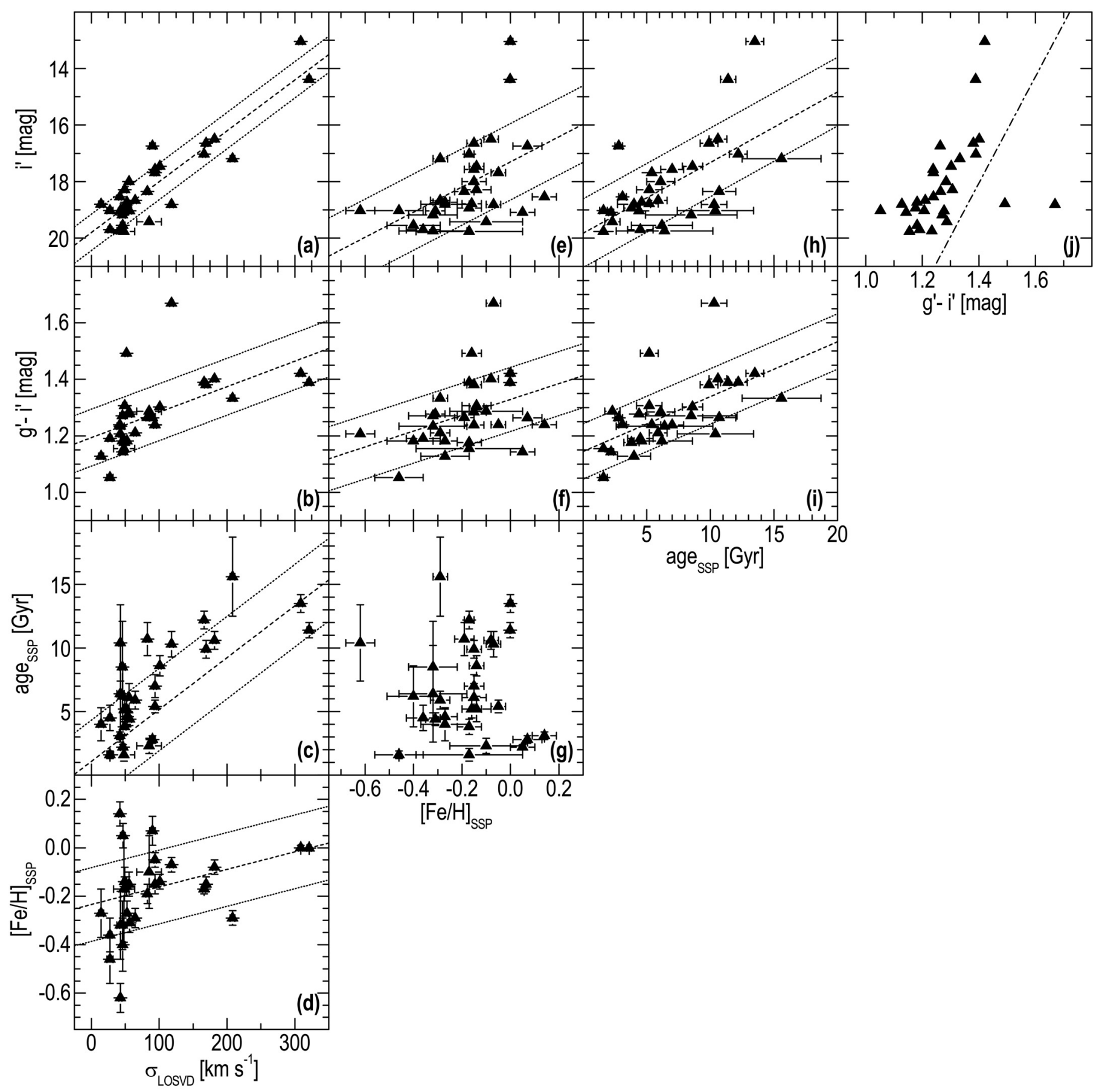

Fig. 11. Scaling relations for the studied galaxy population in the RX J1548.9+0851 system. Dashed lines are linear least squares fits to the data. Dotted lines indicate one-sigma confidence intervals. The dash-dotted line in panel $\mathbf{j}$ ) is the red sequence upper limit as shown in Fig. 3.

dynamical formulae as shown in Table 2 yields a value of $1 / 2 r_{\mathrm{V}}=$ $305 \mathrm{kpc}$, however, classifying RX J1548.9+0851 as fossil. In any case the group is of special interest since it is lacking any other bright galaxies that would be expected in a poor group environment. Multi-object spectroscopy of the RX J1548.9+0851 galaxy population has revealed a luminosity-weighted velocity dispersion of $568 \mathrm{~km} \mathrm{~s}^{-1}$ and a mass of $\sim 2.5 \times 10^{14} M_{\odot}$ for the system. This result confirms previous studies that fossils seem to

proposed by Jones et al. (2003). This temperature limit seems reasonable since the lowest gas temperature of the 15 fossils listed by Mendes de Oliveira et al. (2006) shows a similar value (NGC 6482; Khosroshahi et al. 2004). be very massive (Mendes de Oliveira et al. 2006, 2009; Cypriano et al. 2006; Khosroshahi et al. 2007; Proctor et al. 2011) classifying them as fossil clusters rather than groups inconsistent with the proposed merging scenario. Figure 12 shows mass-to-light ratios and dynamical masses of ordinary poor groups and clusters from Girardi et al. (2002) and 10 fossils from Proctor et al. (2011). Fossils occupy the upper envelope of the distribution which had already been previously discovered by Khosroshahi et al. (2007). The mass-to-light ratio of RX J1548.9+0851 confirms these studies, showing a value of $M / L_{r} \sim 400 M_{\odot} / L_{r, \odot}$.

Khosroshahi et al. (2007) and Proctor et al. (2011) have studied scaling relations of fossils relating X-ray luminosity 


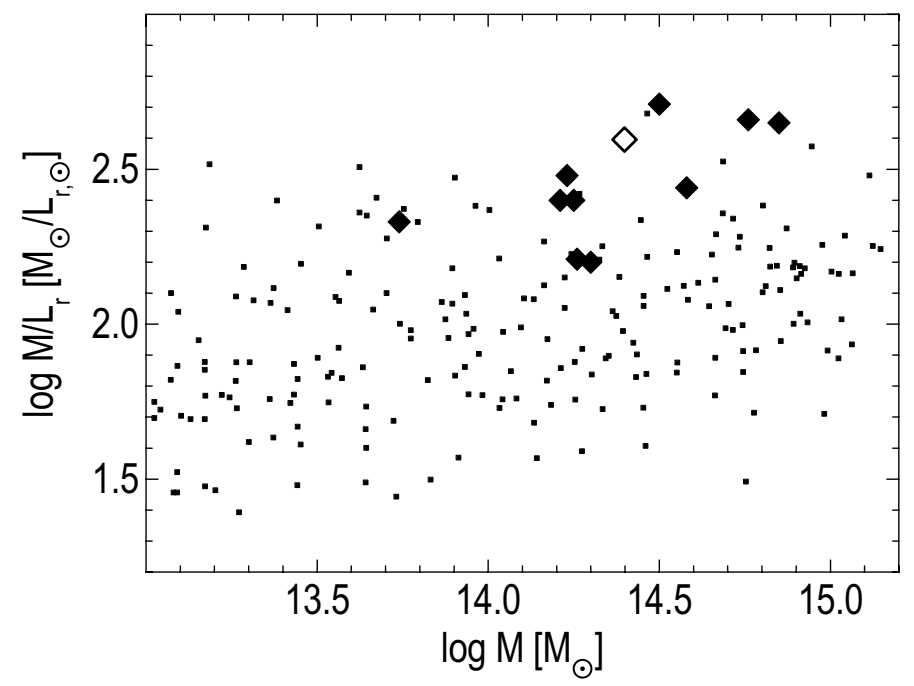

Fig. 12. Mass-to-light ratios plotted against dynamical mass. Black squares are groups and clusters from Girardi et al. (2002) whilst black diamonds are the fossils from Proctor et al. (2011). RX J1548.9+0851 is shown as open diamond.

$L_{\mathrm{X}}$, optical luminosity $L_{r}$, and velocity dispersion $\sigma$. Whilst Khosroshahi et al. (2007) found that, for a given velocity dispersion, fossils are overluminous in X-rays, but comparable to ordinary groups in $L_{\mathrm{r}}$, Proctor et al. (2011) claimed that for a given velocity dispersion, fossils are underluminous in the optical, but otherwise fall on the $L_{X}-\sigma$ relation of groups and clusters. These results led to contrary interpretations, either fossils formed early, building up a cuspy dark matter halo, leading to the excess in X-ray luminosity (Khosroshahi et al. 2007), or they are cluster-like in their masses, but otherwise possess the richness and optical luminosity of relatively poor groups (Proctor et al. 2011). Figure 13 shows the location of the RX J1548.9+0851 system in the $L_{\mathrm{X}}-\sigma, L_{r}-\sigma$, and $L_{\mathrm{X}}-L_{r}$ planes. In all diagrams, RX J1548.9+0851 is found within the distribution of fossils from Proctor et al. (2011), being also clearly underluminous in the optical. Only in the $L_{X}-\sigma$ plane, RX J1548.9+0851 is not as X-ray luminous with respect to other fossils. However, X-ray luminosities are derived from low-signal ROSAT count rates which aren't the best quality data to accurately estimate the overall X-ray luminosity. The present findings on RX J1548.9+0851 support the interpretation from Proctor et al. (2011), leaving the question of what happened to all the missing baryons.

The colour-magnitude diagram of RX J1548.9+0851 reveals a pronounced red sequence and a lack of blue galaxies within the system. In fact, $90 \%$ of all spectroscopically confirmed member galaxies in the inner $300 \mathrm{kpc}$ of the group are found within a narrow band of $\sim 0.3$ mag thickness in colour. Galaxies bluer than this distribution are at least 6 mag fainter than the central elliptical. Moving out to $1 \mathrm{Mpc}$, still $89 \%$ of all spectroscopically confirmed members are found within this stripe, slightly increasing in thickness to $0.4 \mathrm{mag}$. Again, blue galaxies outside this region are $\sim 6$ mag fainter than the central galaxy and constitute of only 4 objects (see Fig. 3). This dominance of red galaxies within the group suggests that most of these galaxies, especially the luminous ones, are old and passively evolving. A similar result was found in the CMDs of the two fossil groups CXGG $095951+0140.8$ and CXGG095951+0212.6 at $z \sim 0.4$ discovered in the Cosmic Evolution Survey (COSMOS) by Pierini et al. (2011). Only 2 members are found above the adopted red sequence upper limit. Interestingly, these 2 galaxies are located in the immediate vicinity of the two brightest ellipticals and show a compact, circular appearance. A similar object has been found around the central elliptical in the evolved NGC 5846 group within the Local Supercluster (Eigenthaler \& Zeilinger 2010) suggesting that these satellites have been tidally stripped by their massive host galaxies.

Symmetric shells are revealed unambiguously along the major axis of the central elliptical. The presence of these symmetric shells indicates a recent minor merger in the centre of the system. In fact, Ebrová et al. (2010) have simulated shell galaxies with the conclusion that a significantly smaller secondary galaxy interacting with the dark matter halo of the primary elliptical can reproduce such observed regular shell systems. In their simulation, a satellite falling radially towards the centre of the primary galaxy is assumed to be instantly ripped apart when approaching the centre of the primary. Its stars subesequently oscillate in the potential of the primary elliptical and produce arc-like structures at the orbit turning points as observed. Thus, being the most massive object in the RX J1548.9+0851 system and exhibiting the observed fine structure, it is very likely that a recent minor merger occured in this system. This would be in agreement with the evolution scenario for fossils as proposed by Dariush et al. (2007) suggesting that these aggregates have assembled most of their mass at early epochs and grow typically by minor mergers afterwards.

Existing studies have revealed steep, increasing luminosity functions for the fossil clusters RX J1340.6+4018 ( $\alpha=-1.6 \pm$ 0.2; Mendes de Oliveira et al. 2009) and RX J1416.4+2315 ( $\alpha=-1.2$ to -1.3 ; Cypriano et al. 2006), consistent with those of other clusters (e.g. Virgo and Coma). On the other hand, a decreasing faint-end slope has been found for the fossil RX J1552.2+2013 ( $\alpha=-0.4$ to -0.8 ; Mendes de Oliveira et al. 2006). Interestingly, this fossil is also the only one in the literature to exhibit a $\mathrm{cD}$ envelope around the central elliptical and a dip in the luminosity function around $M_{r^{\prime}}=-18$. Hence, Mendes de Oliveira et al. (2006) argued that the presence of a $\mathrm{cD}$ envelope around the central elliptical could be related to the lack of faint galaxies and the steepness of the faint-end luminosity function. The RX J1548.9+0851 system studied in this work also revealed a steep, increasing luminosity function, exhibiting high values around $\alpha=-1.4 \pm 0.1$. No lack of faint galaxies, i.e. a pronounced dip around $M_{r^{\prime}}=-18+5 \log h$ has been found. The surface brightness profile of the central elliptical is wellfitted by a pure deVaucouleurs $r^{1 / 4}$ law without any indication of a light excess in the outer galaxy regions, consistent with the suggested scenario highlighted in the previous studies.

Spectra of RX J1548.9+0851 member galaxies have been fitted with SSP models to determine SSP equivalent ages and metallicities of the group galaxy population. The distribution of SSP ages within the system shows a clear spatial segregation. Whilst galaxies exhibiting an old ( $>8 \mathrm{Gyr}$ ) stellar population are confined to an elongated, central distribution, galaxies with young $(<8 \mathrm{Gyr})$ stars are more diffusely distributed and predominantly found in the outer regions of the system. Interestingly, the distribution of old galaxies is also correlated with the orientation of the major axis of the central elliptical. This is in agreement with the work of Sales et al. (2007) who used the Millennium simulation to investigate correlations between the central primary galaxies and the surrounding galaxy populations. They found that red satellites are clearly much more concentrated towards a massive central galaxy than blue ones. They argue that this is presumably due to the gas loss of satellites once they are accreted. The earlier a satellite was accreted, the older, i.e. redder, its stellar population will be. This suggests 


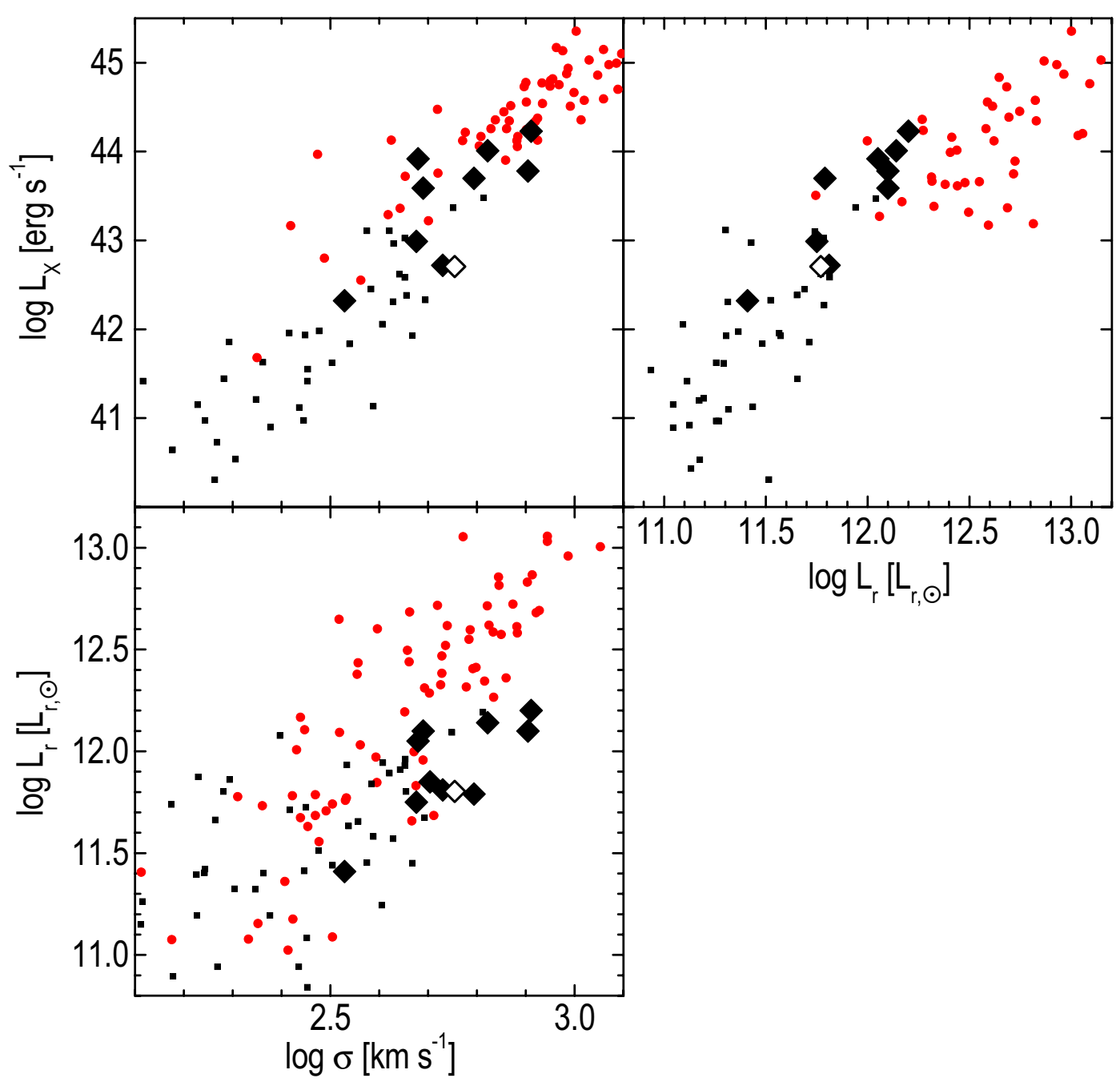

Fig. 13. The RX J1548.9+0851 system in scaling relations of ordinary groups and clusters. Black squares are groups from Osmond \& Ponman (2004) and Girardi et al. (2002) whilst red dots are clusters from Girardi et al. (2002) and Wu et al. (1999). Black diamonds are fossils from Proctor et al. (2011). RX J1548.9+0851 is shown as open diamond.

that the distribution of old stellar population galaxies in the RX J1548.9+0851 system was formed first, likely representing a virialised substructure whereas galaxies with younger stellar populations are not in dynamical equilibrium within the group potential. The simulation also revealed a systematic anisotropy in the spatial distribution of satellites around primaries. It has been established that dark matter haloes are triaxial objects, preferentially exhibiting prolate shapes whose angular momentum is perpendicular to the major-axis of the halo (Hopkins et al. 2005). Simulated satellites show an anti-Holmberg effect, being aligned along the plane perpendicular to the angular momentum axis of the halo. Thus, based on this simulation and assuming that the major axis of a primary is aligned with the major axis of the dark matter halo, an alignment of satellites with the major axis of the central galaxy in fossils is expected. The results presented in Fig. 7 show such an alignment. The measured SSP ages are also correlated with the observed radial velocity distribution. Old galaxies are mainly found at velocities around the two brightest ellipticals BG1 and BG2 which are also the oldest objects in the system. In contrast, young objects show a smooth distribution over all velocities and exhibit a much larger dispersion supporting the highlighted scenario where the old galaxy population assembled first, leaving enough time to virialise around the most massive subhaloes BG1 and BG2.

\section{Conclusion}

We have presented a spectroscopic analysis of the fossil candidate RX J1548.9+0851, selected from the sample of Santos et al. (2007). The system is dominated by two bright ellipticals in the centre, questioning the definition as fossil. Nonetheless, similarities to other fossils studied recently have been found, given that the system is rather comparable to clusters in mass, shows a steep faint-end luminosity function, high mass-to-light ratio, and a central elliptical without $\mathrm{cD}$ envelope. Ages and radial velocities of member galaxies suggest that satellites hosting old stellar populations form a virialised substructure. Shells around the central elliptical indicate the presence of a recent minor merger. Comparing RX J1548.9+0851 with scaling relations from ordinary poor groups and clusters confirm the idea that fossils might simply be normal clusters with the richness of poor groups. The missing baryons could have been expelled from the system or are hidden in the intracluster light.

Acknowledgements. We acknowledge the useful comments from the anonymous referee which helped to improve the paper. P.E. was supported by the University of Vienna in the frame of the Initiative Kolleg (IK) The Cosmic Matter Circuit I033-N. This work is based on observations made with the ESO-VLT at the La Silla Paranal Observatory under programme ID 383.A-0123 and made use of the astronomical data reduction software IRAF which is distributed by the National Optical Astronomy Observatories (NOAO). 
P. Eigenthaler and W. W. Zeilinger: RX J1548.9+0851, a fossil cluster?

\section{References}

Adelman-McCarthy, J. K., Agüeros, M. A., Allam, S. S., et al. 2008, ApJS, 175, 297

Barnes, J. E. 1989, Nature, 338, 123

Biviano, A., Katgert, P., Mazure, A., et al. 1997, A\&A, 321, 84

Cypriano, E. S., Mendes de Oliveira, C. L., \& Sodré, L. Jr. 2006, AJ, 132, 514

Dariush, A., Khosroshahi, H. G., Ponman, T. J., et al. 2007, MNRAS, 382, 433

D’Onghia, E., Sommer-Larsen, J., Romeo, A. D., et al. 2005, ApJ, 630, 109

Ebrová, I., Jungwiert, B., Canalizo, G., et al. 2010, ASPC, 423, 236

Eigenthaler, P., \& Zeilinger, W. W. 2009, AN, 330, 978

Eigenthaler, P., \& Zeilinger, W. W. 2010, A\&A, 511, A12

Evrard, A. E., Metzler, C. A., \& Navarro, J. F. 1996, ApJ, 469, 494

Firth, P., Evstigneeva, E. A., Jones, J. B., et al. 2006, MNRAS, 372, 1856

Girardi, M., Manzato, P., Mezzetti, M., et al. 2002, ApJ, 569, 720

Hartley, W. G., Gazzola, L., Pearce, F. R., et al. 2008, MNRAS, 386, 2015

Hopkins, P. F., Bahcall, N. A., \& Bode, P. 2005, ApJ, 618, 1

Horne, K. 1986, PASP, 98, 609

Jacoby, G. H., Hunter, D. A., \& Christian, C. A. 1984, ApJS, 56, 257

Jones, L. R., Ponman, T. J., Horton, A., et al. 2003, MNRAS, 343, 627

Khosroshahi, H. G., Jones, L. R., \& Ponman, T. J. 2004, MNRAS, 349, 1240

Khosroshahi, H. G., Ponman, T. J., \& Jones, L. R. 2006, MNRAS, 372, 68

Khosroshahi, H. G., Ponman, T. J., \& Jones, L. R. 2007, MNRAS, 377, 595

Koleva, M., Prugniel, P., Bouchard, A., \& Wu, Y. 2009, A\&A, 501, 1269

Kurtz, M. J., \& Mink, D. J. 1998, PASP, 110, 934

Le Borgne, D., Rocca-Volmerange, B., Prugniel, P., et al. 2004, A\&A, 425, 881

Miller, E. D., Rykoff, E., Dupke, R., et al. 2012, ApJ, 747, 94
Mendes de Oliveira, C. L., Cypriano, E. S., \& Sodré, L. Jr. 2006, AJ, 131, 158 Mendes de Oliveira, C. L., Cypriano, E. S., Dupke, R. A., \& Sodré, L. Jr. 2009, AJ, 138, 502

Mukai, K. 1993, Legacy, 3, 21

Navarro, J. F., Frenk, C. S., \& White, S. D. M. 1995, MNRAS, 275, 720

Osmond, J. P. F., \& Ponman, T. J. 2004, MNRAS, 350, 1511

Pierini, D., Giodini, S., Finoguenov, A., et al. 2011, MNRAS, 417, 2927

Ponman, T. J., Allan, D. J., Jones, L. R., et al. 1994, Nature, 369, 462

Proctor, R., Mendes de Oliveira, C. L., Dupke, R. A., et al. 2011, MNRAS, 418, 2054

Prugniel, P., \& Soubiran, C. 2001, A\&A, 369, 1048

Prugniel, P., Soubiran, C., Koleva, M., \& Le Borgne, D. 2007 [arXiv:astro.ph0703658]

Ramella, M., Diaferio, A., Geller, M. J., \& Huchra, J. P. 1994, AJ, 107, 1623

Sales, L. V., Navarro, J. F., Lambas, D. G., et al. 2007, MNRAS, 382, 1901

Santos, W. A., Mendes de Oliveira, C. L., \& Sodré, L. Jr. 2007, AJ, 134, 1551

Schirmer, M., Suyu, S., Schrabback, T., et al. 2010, A\&A, 514, A60

Sodré, L. Jr., Capelato, H. V., Steiner, J. E., \& Mazure, A. 1989, AJ, 97, 1279

Springel, V., White, S. D. M., Jenkins, A., et al. 2005, Nature, 435, 629

Sun, M., Forman, W., Vikhlinin, A., et al. 2004, ApJ, 612, 805

Tonry, J., \& Davis, M. 1979, AJ, 84, 1511

Ulmer, M. P., Adami, C., Covone, G., et al. 2005, ApJ, 624, 124

Vikhlinin, A., McNamara, B. R., Hornstrup, A., et al. 1999, ApJ, 520, 1

Voges, W., Aschenbach, B., Boller, T., et al. 1999, A\&A, 349, 389

Wu, X., Xue, Y., \& Fang, L. 1999, ApJ, 524, 22

Yoshioka, T., Furuzawa, A., Takahashi, S., et al. 2004, AdSpR, 34, 2525 